International Research Journal of Engineering, IT \& Scientific Research
Available online at https://sloap.org/journals/index.php/irjeis/
Vol. 5 No. 2, March 2019, pages: 1 28
ISSN: 2454-2261
https://doi.org/10.21744/irjeis.v5n2.607

\title{
Stability of Thin Laminated Decks Plates under Plane Compressive Loading
}

Osama Mohammed Elmardi Suleiman ${ }^{\text {a }}$ Mahmoud Yassin Osman ${ }^{\text {b }}$

Tagelsir Hassan $^{c}$

Article history:

Received: 18 September 2018

Accepted: 31 January 2019

Published: 21 March 2019

\section{Keywords:}

biaxial buckling;

classical laminated plate theory;

finite element;

composite laminated decks

plates;

fortran program;

\begin{abstract}
It was found that symmetric laminates are stiffer than the anti - symmetric one due to coupling between bending and stretching which decreases the buckling loads of symmetric laminates. The buckling load increases with increasing aspect ratio, and decreases with increase in modulus ratio. The buckling load will remain the same even when the lamination order is reversed. The buckling load increases with the mode number but at different rates depending on the type of end support. It is also observed that as the mode number increases, the plate needs additional support.
\end{abstract}

2454-2261 ${ }^{\odot}$ Copyright 2019. The Author. This is an open-access article under the CC BY-SA license (https://creativecommons.org/licenses/by-sa/4.0/) All rights reserved.

\section{Author correspondence:}

Osama Mohammed Elmardi Suleiman,

Professor in Department of Mechanical Engineering, Faculty of Engineering and Technology,

Nile Valley University Atbara, Sudan.

Email address: osamamm64@gmail.com

\section{Introduction}

The members and structures composed of laminated composite material are usually very thin, and hence more prone to buckling. Buckling phenomenon is critically dangerous to structural components because the buckling of composite plates usually occurs at a lower applied stress and generates large deformations. This led to a focus on the study of buckling behavior in composite materials. General introductions to the buckling of elastic structures and of laminated plates can be found in (Chai \& Khong, 1993), (Narita \& Fukushi, 1996), (Turvey \& Marshal, 1995), (Singer et al., 1998, 2002), (Reddy, 2004), and (Berthelot, 1999). However, these available curves and data are restricted to idealized loading, namely, uniaxial or biaxial uniform compression.

a Professor in Department of Mechanical Engineering, Faculty of Engineering and Technology, Nile Valley University Atbara, Sudan

b Department of Mechanical Engineering, Faculty of Engineering, Kassala University, Kassala, Kassala State, Sudan

c Department of Mechanical Engineering, Faculty of Engineering, Omdurman Islamic University, Omdurman, Khartoum State, Sudan 
Due to the importance of buckling considerations, there is an overwhelming number of investigations available in which corresponding stability problems are considered by a wide variety of analysis methods which may be of a closed - form analytical nature or may be sorted into the class of semi - analytical or purely numerical analysis method.

Closed - form exact solutions for the buckling problem of rectangular composite plates are available only for limited combinations of boundary conditions and laminated schemes. These include cross - ply symmetric and angle - ply anti - symmetric rectangular laminates with at least two opposite edges simply supported, and similar plates with two opposite edges clamped but free to deflect (i.e. guided clamp) or with one edge simply supported and the opposite edge with a guided clamp. Most of the exact solutions discussed in the monographs of (Whitney, 1987), who developed an exact solution for critical buckling of solid rectangular orthotropic plates with all edges simply supported, and of (Reddy, 1997, 1984), ( Reddy \& Phan, 1985), (Reddy \& Khdeir, 1989) and (Leissa \& Kang, 2002) and that of (Iyengar,1988). Bao et al., (1997), developed an exact solution for two edges simply supported and two edges clamped, and Robinson (1955), who developed an exact solution for the critical buckling stress of an orthotropic sandwich plate with all edges simply supported.

For all other configurations, for which only approximated results are available, several semi - analytical and numerical techniques have been developed. The Rayleigh - Ritz method (Iyengar, 1988), the finite strip method (FSM) (Chai \& Khong, 1993) and (Dawe \& Wang, 1995), the element free Galerkin method (EFG) (Liu et al., 2002), the differential quadrature technique (Bert \& Malik, 1997), the moving least square differential quadrature method (Huang \& Li, 2004) and the most extensively used finite element method (FEM) (Kim \& Hoa, 1995), are the most common ones.

The Kantorovich method (KM) (Shufrin et al., 2007), which is a different and in most cases advantageous semi analytical method, combines a variation approach of closed - form solutions and an iterative procedure. The method assumes a solution in the form of a sum of products of functions in one direction and functions in the other direction. Then, by assuming the function in one direction, the variation problem of the plate reduces to a set of ordinary differential equations. In the case of buckling analysis, the variation problem reduces to an ordinary differential eigenvalue and Eigen function problem. The solution of the resulting problem is an approximate one, and its accuracy depends on the assumed functions in the first direction. The extended Kantorovich method (EKM), which was proposed by Kerr (1969), is the starting point for an iterative procedure, where the solution obtained in one direction is used as the assumed functions in the second direction. After repeating this process several times, convergence is obtained. The single term extended Kantorovich method was employed for a buckling analysis of rectangular plates by several researches. Eienberger \& Alexandrov (2003), used the method for the buckling analysis of isotropic plates with variable thickness. Shufrin \& Eienberger (2005), extended the solution to thick plates with constant and variable thickness using the first and higher order shear deformation theories. Ungbhakorn \& Singhatanadgid (2006), extended the solution to buckling of symmetrically cross - ply laminated rectangular plates. The multi - term formulation of the extended Kantorovich approach to the simplest samples of rectangular isotropic plates was presented by Yuan \& Jin (1998), this study showed that the additional terms in the expansion can be used in order to improve the solution.

March \& Smith (1945), found an approximate solution for all edges clamped. Also, Chang et al., (1962), developed approximate solution to the buckling of rectangular orthotropic sandwich plate with two edges simply supported and two edges clamped or all edges clamped using the March - Erickson method and an energy technique. Jiang et al., (1977), developed solutions for local buckling of rectangular orthotropic hat - stiffened plates with edges parallel to the stiffeners were simply supported or clamped and edges parallel to the stiffeners were free, and Smith (1990), presented solutions bounding the local buckling of hat stiffened plates by considering the section between stiffeners as simply supported or clamped plates.

Many authors have used finite element method to predict accurate in - plane stress distribution which is then used to solve the buckling problem. Zienkiewicz (1977) and Cook (1981), have clearly presented an approach for finding the buckling strength of plates by first solving the linear elastic problem for a reference load and then the eigenvalue problem for the smallest eigenvalue which then multiplied by the reference load gives the critical buckling load of the structure. An excellent review of the development of plate finite elements during the past 35 years was presented by (Yang et al., 2000).

Many buckling analyses of composite plates available in the literature are usually realized parallel with the vibration analyses, and are based on two - dimensional plate theories which may be classified as classical and shear deformable ones. Classical plate theories (CPT) do not take into account the shear deformation effects and over predict the critical buckling loads for thicker composite plates, and even for thin ones with a higher anisotropy. Most 
of the shear deformable plate theories are usually based on a displacement field assumption with five unknown displacement components. As three of these components corresponded to the ones in CPT, the additional ones are multiplied by a certain function of thickness coordinate and added to the displacements field of CPT in order to take into account the shear deformation effects.

Taking these functions as linear and cubic forms leads to the so - called uniform or Mindlin shear deformable plate theory (USDPT) (Mindlin, 1951), and parabolic shear deformable plate theories (PSDPT) (Reddy, 1984) respectively. Different forms were also employed such as hyperbolic shear deformable plate theory (HSDPT) (Soladatos, 1992), and trigonometric or sine functions shear deformable plate theory (TSDPT) (Touratier, 1991). Since these types of shear deformation theories do not satisfy the continuity conditions among many layers of the composite structures, the zig - zag type of the plate theories introduced by Di Sciuva (1987) and Cho \& Parmerter (1993), in order to consider interlaminar stress continuities. Recently, Karama et al., (2003), proposed a new exponential function \{i.e. exponential shear deformable plate theory (ESDPT) $\}$ in the displacement field of the composite laminated structures for the representation of the shear stress distribution along the thickness of the composite structures and compared their result for static and dynamic problem of the composite beams with the sine model.

Within the classical lamination theory, Jones (1973) presented a closed - form solution for the buckling problem of cross - ply laminated plates with simply supported boundary conditions. In the case of multi - layered plates subjected to various boundary conditions which are different from simply supported boundary conditions at all of their four edges, the governing equations of the buckling of the composite plates do not admit an exact solution, except for some special arrangements of laminated plates. Thus, for the solution of these types of problems, different analytical and / or numerical methods are employed by various researchers. Narita \& Leissa (1989), applied the Ritz method with the displacement components assumed as the double series of trigonometric functions for the buckling problem of generally symmetric laminated composite rectangular plates with simply supported boundary conditions at all their edges. They investigated the critical buckling loads for five different types of loading conditions which are uniaxial compression (UA - C), biaxial compression (BA - C), biaxial compression - tension (BA - CT), and positive and negative shear loadings.

The higher - order shear deformation theories can yield more accurate inter - laminate stress distributions. The introduction of cubic variation of displacement also avoids the need for shear correction displacement. To achieve a reliable analysis and safe design, the proposals and developments of models using higher order shear deformation theories have been considered. Lo et al., (1977), reviewed the pioneering work on the field and formulated a theory which accounts for the effects of transverse shear deformation, transverse strain and non - linear distribution of the in - plane displacements with respect to the thickness coordinate. Third - order theories have been proposed by Reddy (1993); Librescu (1975), Schmidt (1977); Murthy (1981); Levinson (1980); Seide (1980); Bhimaraddi et al., (1984); Mallikarjuna \& Kant (1993); Kant \& Pandya (1988); and Phan \& Reddy (1985). Pioneering work and overviews in the field covering closed - form solutions and finite element models can be found in (Reddy, 1980); (Noor \& Burton, 1990); ( Bert, 1984); ( Kant \& Kommineni, 1984); and (Reddy and Robbins, 1994).

For the buckling analysis of the cross - ply laminated plates subjected to simply supported boundary conditions at their opposite two edges and different boundary conditions at the remaining ones (Khdeir, 1989) and (Reddy \& Khdeir, 1989), used a parabolic shear deformation theory and applied the state - space technique. Hadian \& Nayfeh (1993), on the basis of the same theory and for the same type of problem, needed to modify the technique due to ill conditioning problems encountered especially for thin and moderately thick plates. The buckling analyses of completely simply supported cross - ply laminated plates were presented by Fares \& Zenkour (1999), who added a non - homogeneity coefficient in the material stiffnesses within various plate theories, and by Matsunaga (2000), who employed a global higher order plate theory. Gilat el al., (2001), also investigated the same type of problem on the basic of a global - local plate theory where the displacement field is composed of global and local contributions, such that the requirement of the continuity conditions and delamination effects can be incorporated into formulation.

Many investigations have been reported for static and stability analysis of composite laminates using different traditional methods. Pagano (1970), developed an exact three - dimensional (3 - D) elasticity solution for static analysis of rectangular bi - directional composites and sandwich plates. (Noor, 1975) presented a solution for stability of multi - layered composite plates based on $3-\mathrm{D}$ elasticity theory by solving equations with finite difference method. Also, 3 - D elasticity solutions are presented by Gu \& Chattopadhyay (2000), for the buckling of simply supported orthotropic composite plates. When the problem is reduced from a three - dimensional one ( $3-\mathrm{D})$ to a two-dimensional case to contemplate more efficiently the computational analysis of plate composite structures,

Suleiman, O. M. E., Osman, M. Y., \& Hassan, T. (2019). Stability of thin laminated decks plates under plane compressive loading. International Research Journal of Engineering, IT \& Scientific Research, 5(2), 1-28. 
the displacement based theories and the corresponding finite element models receive the most attention (Reddy, 1993).

Bifurcation buckling of laminated structures has been investigated by many researchers without considering the flatness before buckling (Leissa, 1986). This point was first clarified for laminated composite plates for some boundary conditions and for some lamina configurations by (Leissa, 1986). Qatu \& Leissa (1993), applied this result to identify true buckling behavior of composite plates.

It is important to recognize that, with the advent of composite media, certain new material imperfections can be found in composite structures in addition to the better - known imperfections that one finds in metallic structures. Thus, broken fibers, delaminated regions, cracks in the matrix material, as well as holes, foreign inclusions and small voids constitute material and structural imperfections that can exist in composite structures. Imperfections have always existed and their effect on the structural response of a system has been very significant in many cases. These imperfections can be classified into two broad categories: initial geometrical imperfections and material or constructional imperfections.

The first category includes geometrical imperfections in the structural configuration (such as a local out of roundness of a circular cylindrical shell, which makes the cylindrical shell non - circular; a small initial curvature in a flat plate or rod, which makes the structure non - flat, etc.), as well as imperfections in the loading mechanisms (such as load eccentricities; an axially loaded column is loaded at one end in such a manner that a bending moment exists at that end. The second class of imperfections is equally important, but has not received as much attentions as the first class; especially as far as its effect on the buckling response characteristics is concerned. For metallic materials, one can find several studies which deal with the effect of material imperfections on the fatigue life of the structural component. Moreover, there exist a number of investigations that deal with the effect of cut - outs and holes on the stress and deformation response of thin plates. Another material imperfection is the rigid inclusion. The effect of rigid inclusions on the stress field of the medium in the neighborhood of the inclusion has received limited attention. The interested reader is referred to the bibliography of Professor (Naruoka, 1981).

In the present study, the composite media are assumed free of imperfections i.e. initial geometrical imperfections due to initial distortion of the structure, and material and / or constructional imperfections such as broken fibers, delaminated regions, cracks in the matrix material, foreign inclusions and small voids which are due to inconvenient selection of fibers / matrix materials and manufacturing defects. Therefore, the fibers and matrix are assumed perfectly bonded.

\section{Mathematical Formulations}

\section{1) Introduction}

The choice of the coordinate system is of critical importance for laminated plates. This is because plates with rectangular orthotropic could be set on rectangular, triangular, circular or other boundaries. Composite materials with rectangular orthotropic are the most popular, mainly because of their ease in design and manufacturing. The equations that follow are developed for materials with rectangular orthotropic.

Figure 1 shows the geometry of a plate with rectangular orthotropic drawn in the Cartesian coordinates X, Y, and Z or 1,2, and 3. The parameters used in such a plate are: (1) the length in the X-direction, (a); (2) the length in the Y direction (i.e. breadth), (b); and (3) the length in the $\mathrm{Z}$ - direction (i.e. thickness), (h). 


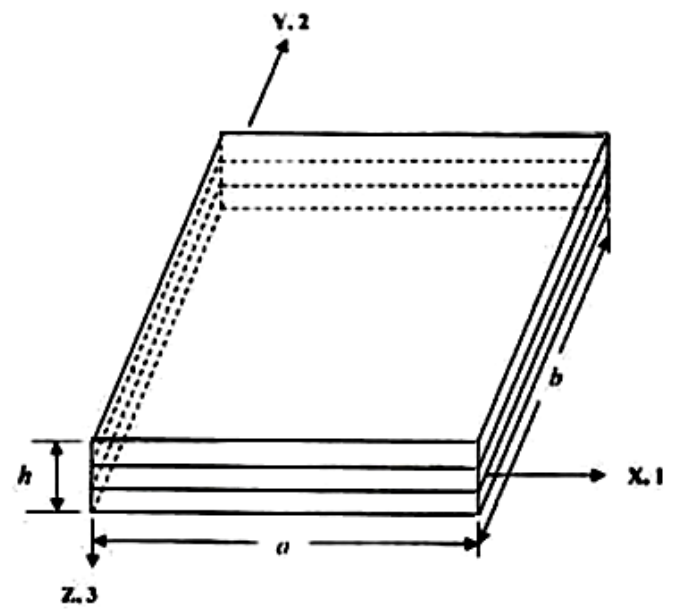

Figure 1. The geometry of a laminated composite plate

\section{2) Fundamental Equations of Elasticity}

Classical laminated plate theory (CLPT) is selected to formulate the problem. Consider a thin plate of length a, breadth $b$, and thickness $h$ as shown in Figure 2(a), subjected to in - plane loads Rx, Ry and Rxy as shown in Figure 2(b). The in - plane displacements $\boldsymbol{u}(\boldsymbol{x}, \boldsymbol{y}, \boldsymbol{z})$ and $\boldsymbol{v}(\boldsymbol{x}, \boldsymbol{y}, \boldsymbol{z})$ can be expressed in terms of the out of plane displacement $\boldsymbol{w}(\boldsymbol{x}, \boldsymbol{y})$ as shown below:

The displacements are:

$$
\left.\begin{array}{l}
u(x, y, z)=u_{o}(x, y)-z \frac{\partial w}{\partial x} \\
v(x, y, z)=v_{o}(x, y)-z \frac{\partial w}{\partial y} \\
w(x, y, z)=w_{o}(x, y)
\end{array}\right\}
$$

Where $u_{o}, v_{o}$ and $w_{o}$ are mid - plane displacements in the direction of the $x, y$ and $z$ axes respectively; $z$ is the perpendicular distance from mid - plane to the layer plane.

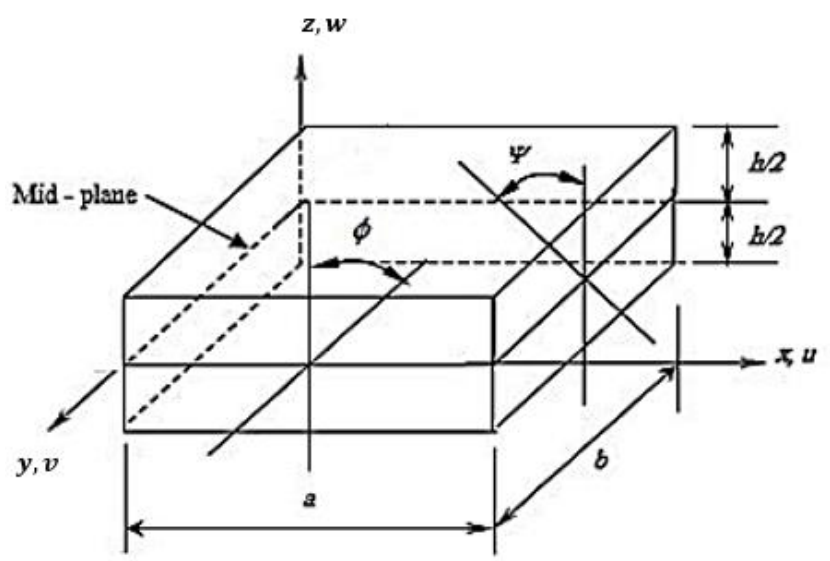

( a )

Suleiman, O. M. E., Osman, M. Y., \& Hassan, T. (2019). Stability of thin laminated decks plates under plane compressive loading. International Research Journal of Engineering, IT \& Scientific Research, 5(2), 1-28. 


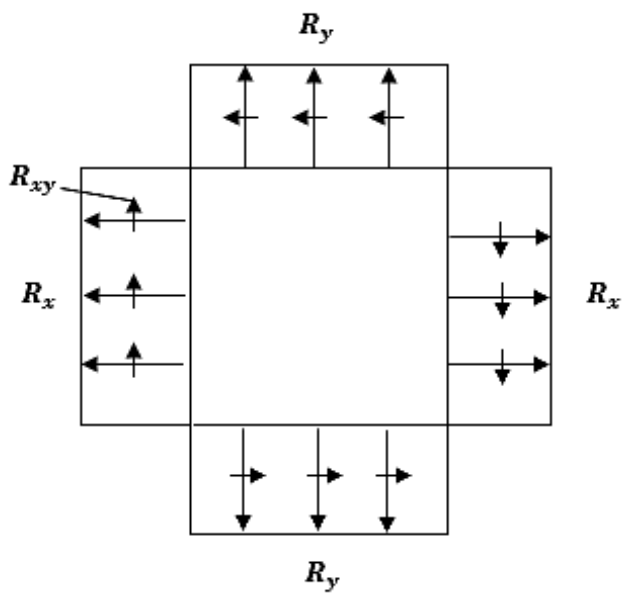

( b )

Figure 2. A plate showing dimensions and deformations

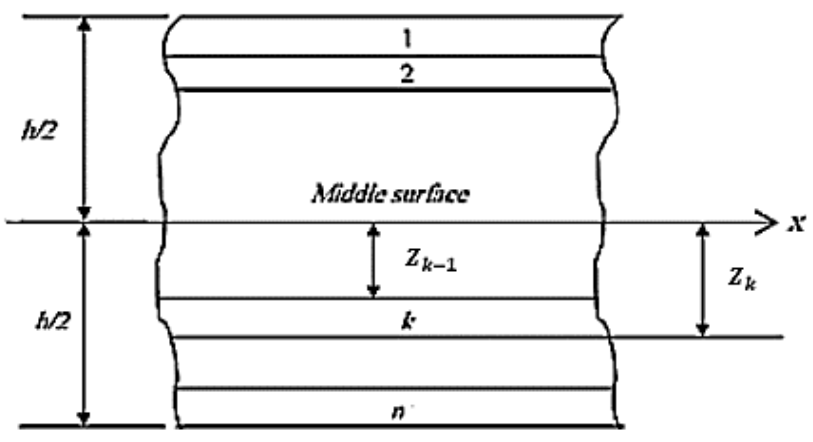

Figure 3. Geometry of an n-layered laminate

The plate shown in Figure 2(a) is constructed of an arbitrary number of orthotropic layers bonded together as in Figure 3.

\section{The strains are:}

The virtual strains:

$$
\left.\begin{array}{l}
\epsilon_{x}=\frac{\partial u_{o}}{\partial x}-z \frac{\partial^{2} w}{\partial x^{2}}+\frac{1}{2}\left(\frac{\partial w}{\partial x}\right)^{2} \\
\epsilon_{y}=\frac{\partial v_{o}}{\partial y}-z \frac{\partial^{2} w}{\partial y^{2}}+\frac{1}{2}\left(\frac{\partial w}{\partial y}\right)^{2} \\
\gamma=\frac{\partial v_{o}}{\partial x}+\frac{\partial u_{o}}{\partial y}-2 z \frac{\partial^{2} w}{\partial x \partial y}+\left(\frac{\partial w}{\partial x}\right)\left(\frac{\partial w}{\partial y}\right)
\end{array}\right\}
$$

$$
\begin{aligned}
\delta \epsilon_{x}= & \frac{\partial}{\partial x} \delta u_{o}-z \frac{\partial^{2}}{\partial x^{2}} \delta w+\frac{\partial w}{\partial x} \frac{\partial}{\partial x} \delta w \\
\delta \epsilon_{y}= & \frac{\partial}{\partial y} \delta v_{o}-z \frac{\partial^{2}}{\partial y^{2}} \delta w+\frac{\partial w}{\partial y} \frac{\partial}{\partial y} \delta w \\
\delta \gamma= & \frac{\partial}{\partial x} \delta v_{o}+\frac{\partial}{\partial y} \delta u_{o}-2 z \frac{\partial^{2}}{\partial x \partial y} \delta w \\
& +\frac{\partial w}{\partial x} \frac{\partial}{\partial y} \delta w+\frac{\partial}{\partial x} \delta w \frac{\partial w}{\partial y}
\end{aligned}
$$




\section{The virtual strain energy:}

$$
\delta U=\int_{V} \delta \epsilon^{T} \sigma d V
$$

But,

$$
\sigma=C \epsilon
$$

Where,

$$
C=C_{i j}(i, j=1,2,6)
$$

$$
\therefore \delta U=\int_{V} \delta \epsilon^{T} C \delta \epsilon d V
$$

If we neglect the in-plane displacements $u_{o}$ and $v_{o}$ and considering only the linear terms in the strain displacement equations, we write:

$$
\delta \epsilon=-z\left|\begin{array}{c}
\frac{\partial^{2}}{\partial x^{2}} \\
\frac{\partial^{2}}{\partial y^{2}} \\
2 \frac{\partial^{2}}{\partial x \partial y}
\end{array}\right| \delta w
$$

\section{Materials and Methods}

\section{The Numerical Method}

The finite element is used in this analysis as a numerical method to predict the buckling loads and shape modes of buckling of laminated rectangular plates (Osama Mohammed Elmardi Suleiman, March 2016, August 2016). In this method of analysis, four - noded type of elements is chosen. These elements are the four - noded bilinear rectangular elements of a plate. Each element has three degrees of freedom at each node. The degrees of freedom are the lateral displacement $(w)$, and the rotations $(\phi)$ and $(\psi)$ about the $(X)$ and $(Y)$ axes respectively.

For an $n$ noded element, and 3 degrees of freedom at each node.

Now express $w$ in terms of the shape functions $N$ and noded displacements $a^{e}$, equation (6) can be written as:

Where,

$$
\delta \epsilon=-z B \delta a^{e}
$$

$$
B^{T}=\left[\begin{array}{lll}
\frac{\partial^{2} N_{i}}{\partial x^{2}} & \frac{\partial^{2} N_{i}}{\partial y^{2}} & 2 \frac{\partial^{2} N_{i}}{\partial x \partial y}
\end{array}\right]
$$

and

$$
N_{i} a_{i}^{e}=\left[w_{i}\right] \quad i=1, n
$$

The stress - strain relation is:

$$
\sigma=C \epsilon
$$

Where $C$ are the material properties which could be written as follows:

Where $V$ denotes volume.

$$
\begin{gathered}
C=\left[\begin{array}{lll}
C_{11} & C_{12} & C_{16} \\
C_{12} & C_{22} & C_{26} \\
C_{16} & C_{26} & C_{66}
\end{array}\right] \\
\delta U=\int_{V}\left(B \delta a^{e}\right)^{T}\left(C z^{2}\right) B a^{e} d V
\end{gathered}
$$

$$
\delta U=\delta a^{e T} \int_{V} B^{T} D B a^{e} d x d y=\delta a^{e T} K^{e} a^{e}
$$

Suleiman, O. M. E., Osman, M. Y., \& Hassan, T. (2019). Stability of thin laminated decks plates under plane compressive loading. International Research Journal of Engineering, IT \& Scientific Research, 5(2), 1-28. 
Where $D_{i j}=\sum_{k=1}^{n} \int_{Z_{k-1}}^{Z_{k}} C_{i j} Z^{2} d Z$ is the bending stiffness, and $K^{e}$ is the element stiffness matrix which could be written as follows:

$$
K^{e}=\int B^{T} D B d x d y
$$

The virtual work done by external forces can be expressed as follows: Refer to Figure 4 .

Denoting the nonlinear part of strain by $\delta \epsilon^{\prime}$

$$
\delta W=\iint \delta \epsilon^{\prime T} \sigma^{\prime} d V=\int \delta \epsilon^{\prime T} N d x d y
$$

Where

$$
\begin{aligned}
& N^{T}=\left[N_{x} N_{y} N_{x y}\right]=\left[\sigma_{x} \sigma_{y} \tau\right] d Z \\
& \delta \epsilon^{\prime}=\left[\begin{array}{c}
\delta \epsilon_{x} \\
\delta \epsilon_{y} \\
\delta \gamma
\end{array}\right]=\left[\begin{array}{cc}
\frac{\partial}{\partial x} \delta w & 0 \\
0 & \frac{\partial}{\partial y} \delta w \\
\frac{\partial}{\partial y} \delta w & \frac{\partial}{\partial x} \delta w
\end{array}\right]\left[\begin{array}{l}
\frac{\partial w}{\partial x} \\
\frac{\partial w}{\partial y}
\end{array}\right]
\end{aligned}
$$

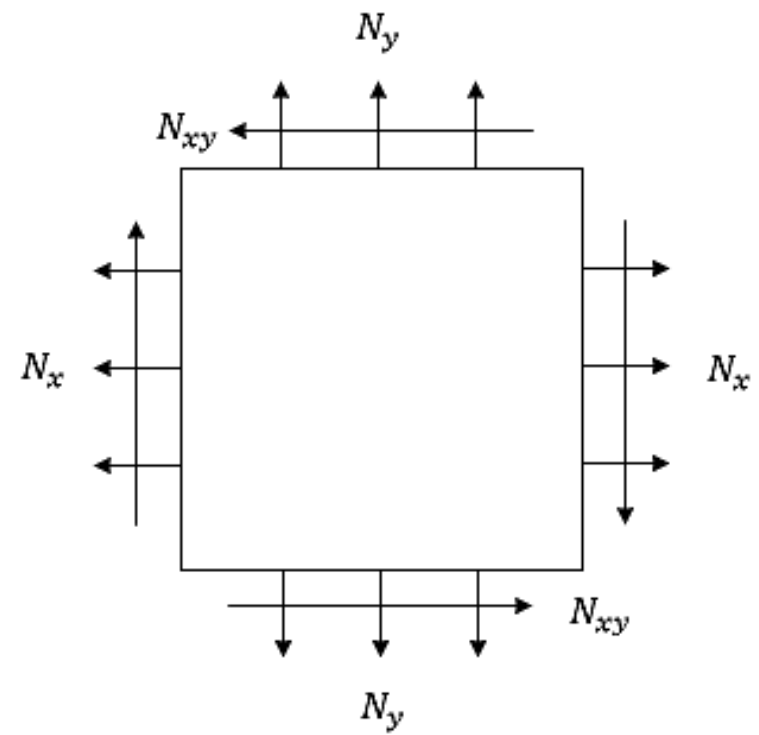

Figure 4. External forces acting on an element

Hence,

$$
\delta W=\iint\left[\begin{array}{l}
\frac{\partial w}{\partial x} \\
\frac{\partial w}{\partial y}
\end{array}\right]^{T}\left[\begin{array}{ccc}
\frac{\partial}{\partial x} \delta w & 0 & \frac{\partial}{\partial y} \delta w \\
0 & \frac{\partial}{\partial y} \delta w & \frac{\partial}{\partial x} \delta w
\end{array}\right]\left[\begin{array}{c}
N_{x} \\
N_{y} \\
N_{x y}
\end{array}\right] d x d y
$$

This can be written as:

$$
\delta W=\iint\left[\begin{array}{l}
\frac{\partial}{\partial x} \delta w \\
\frac{\partial}{\partial y} \delta w
\end{array}\right]^{T}\left[\begin{array}{cc}
N_{x} & N_{x y} \\
N_{x y} & N_{y}
\end{array}\right]\left[\begin{array}{l}
\frac{\partial w}{\partial x} \\
\frac{\partial w}{\partial y}
\end{array}\right] d x d y
$$


Now $w=N_{i} a_{i}^{e}$

$$
\delta W=\delta a^{e T} \iint\left[\begin{array}{l}
\frac{\partial N_{i}}{\partial x} \\
\frac{\partial N_{i}}{\partial y}
\end{array}\right]^{T}\left[\begin{array}{cc}
N_{x} & N_{x y} \\
N_{x y} & N_{y}
\end{array}\right]\left[\begin{array}{c}
\frac{\partial N_{i}}{\partial x} \\
\frac{\partial N_{i}}{\partial y}
\end{array}\right] a^{e} d x d y
$$

Substitute $P_{x}=-N_{x}, P_{y}=-N_{y}, P_{x y}=-N_{x y}$

$$
\delta W=-\delta a^{e T} \iint\left[\begin{array}{c}
\frac{\partial N_{i}}{\partial x} \\
\frac{\partial N_{i}}{\partial y}
\end{array}\right]^{T}\left[\begin{array}{cc}
P_{x} & P_{x y} \\
P_{x y} & P_{y}
\end{array}\right]\left[\begin{array}{c}
\frac{\partial N_{i}}{\partial x} \\
\frac{\partial N_{i}}{\partial y}
\end{array}\right] a^{e} d x d y
$$

Therefore, equation (15) could be written in the following form:

Where,

$$
\delta W=-\delta a^{e T} K^{D} a^{e}
$$

$$
K^{D}=\iint\left[\begin{array}{c}
\frac{\partial N_{i}}{\partial x} \\
\frac{\partial N_{i}}{\partial y}
\end{array}\right]^{T}\left[\begin{array}{cc}
P_{x} & P_{x y} \\
P_{x y} & P_{y}
\end{array}\right]\left[\begin{array}{c}
\frac{\partial N_{i}}{\partial x} \\
\frac{\partial N_{i}}{\partial y}
\end{array}\right] d x d y
$$

$K^{D}$ is the differential stiffness matrix known also as geometric stiffness matrix, initial stress matrix, and initial load matrix.

The total energy:

$$
\delta U+\delta W=0
$$

Since $\delta a^{e}$ is an arbitrary displacement which is not zero, then

$$
K^{e} a^{e}-K^{D} a^{e}=0
$$

Now let us compute the elements stiffness and the differential matrices.

$$
\begin{gathered}
K^{e}=\iint B^{T} D B d x d y \\
K^{e}=\iint\left[\begin{array}{c}
\frac{\partial^{2} N_{i}}{\partial x^{2}} \\
\frac{\partial^{2} N_{i}}{\partial y^{2}} \\
2 \frac{\partial^{2} N_{i}}{\partial x \partial y}
\end{array}\right]^{T}\left[\begin{array}{lll}
D_{11} & D_{12} & D_{16} \\
D_{12} & D_{22} & D_{26} \\
D_{16} & D_{26} & D_{66}
\end{array}\right]\left[\begin{array}{c}
\frac{\partial^{2} N_{i}}{\partial x^{2}} \\
\frac{\partial^{2} N_{i}}{\partial y^{2}} \\
2 \frac{\partial^{2} N_{i}}{\partial x \partial y}
\end{array}\right] d x d y
\end{gathered}
$$

The elements stiffness matrix can be expressed as follows:

$$
\begin{gathered}
K_{i j}^{e}=\iint\left[D_{11} \frac{\partial^{2} N_{i}}{\partial x^{2}} \frac{\partial^{2} N_{j}}{\partial x^{2}}+D_{12}\left(\frac{\partial^{2} N_{i}}{\partial y^{2}} \frac{\partial^{2} N_{j}}{\partial x^{2}}+\frac{\partial^{2} N_{i}}{\partial x^{2}} \frac{\partial^{2} N_{j}}{\partial y^{2}}\right)+2 D_{16}\left(\frac{\partial^{2} N_{i}}{\partial x \partial y} \frac{\partial^{2} N_{j}}{\partial x^{2}}+\frac{\partial^{2} N_{i}}{\partial x^{2}} \frac{\partial^{2} N_{j}}{\partial x \partial y}\right)+D_{22} \frac{\partial^{2} N_{i}}{\partial y^{2}} \frac{\partial^{2} N_{j}}{\partial y^{2}}\right. \\
\left.+2 D_{26}\left(\frac{\partial^{2} N_{i}}{\partial x \partial y} \frac{\partial^{2} N_{j}}{\partial y^{2}}+\frac{\partial^{2} N_{i}}{\partial y^{2}} \frac{\partial^{2} N_{j}}{\partial x \partial y}\right)+4 D_{66} \frac{\partial^{2} N_{i}}{\partial x \partial y} \frac{\partial^{2} N_{j}}{\partial x \partial y}\right] d x d y
\end{gathered}
$$

The elements differential stiffness matrix can be expressed as follows;

$$
K_{i j}^{D}=\iint\left[P_{x} \frac{\partial N_{i}}{\partial x} \frac{\partial N_{j}}{\partial x}+P_{x y}\left(\frac{\partial N_{i}}{\partial y} \frac{\partial N_{j}}{\partial x}+\frac{\partial N_{i}}{\partial x} \frac{\partial N_{j}}{\partial y}\right)+P_{y} \frac{\partial N_{i}}{\partial y} \frac{\partial N_{j}}{\partial y}\right] d x d y
$$

Suleiman, O. M. E., Osman, M. Y., \& Hassan, T. (2019). Stability of thin laminated decks plates under plane compressive loading. International Research Journal of Engineering, IT \& Scientific Research, 5(2), 1-28. 
The shape local co - ordinate for a 4 - noded element is shown below in Figure 5.

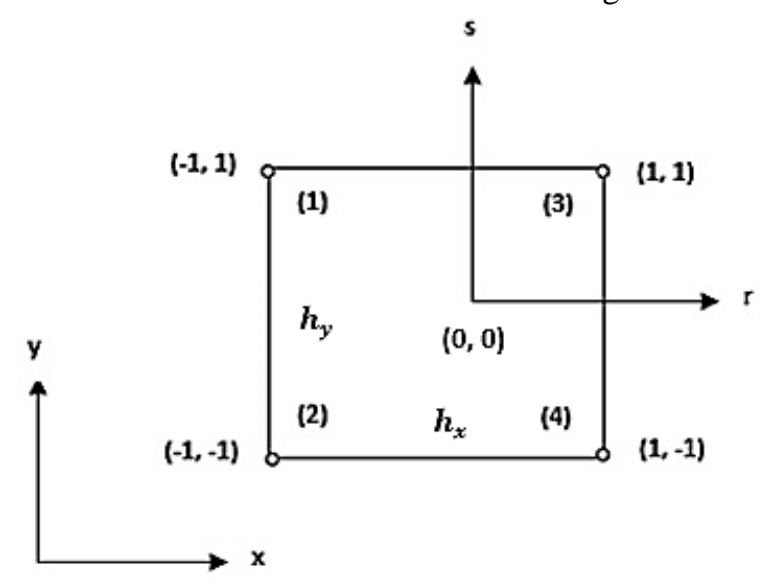

Figure 5. A four noded element with local and global co - ordinates

The shape functions for the 4 - noded element expressed in global co - ordinates $(x, y)$ are as follows:

$$
\begin{aligned}
& w=N_{1} w_{1}+N_{2} \phi_{1}+N_{3} \psi_{1}+N_{4} w_{2}+N_{5} \phi_{2}+N_{6} \psi_{2} \\
& +N_{7} w_{3}+N_{8} \phi_{3}+N_{9} \psi_{3}+N_{10} w_{4}+N_{11} \phi_{4}+N_{12} \psi_{4}
\end{aligned}
$$

Where,

$$
\phi=\frac{\partial w}{\partial x}, \quad \psi=\frac{\partial w}{\partial y}
$$

The shape functions in local co - ordinates are as follows:

$$
\begin{gathered}
N_{i}=a_{i 1}+a_{i 2} r+a_{i 3} s+a_{i 4} r^{2}+a_{i 5} r s+a_{i 6} s^{2}+a_{i 7} r^{3}+a_{i 8} r^{2} s+a_{i 9} r s^{2} \\
+a_{i 10} s^{3}+a_{i 11} r^{3} s+a_{i 12} r s^{3} \\
N_{j}=a_{j 1}+a_{j 2} r+a_{j 3} s+a_{j 4} r^{2}+a_{j 5} r s+a_{j 6} s^{2}+a_{j 7} r^{3}+a_{j 8} r^{2} s+a_{j 9} r s^{2} \\
+a_{j 10} s^{3}+a_{j 11} r^{3} s+a_{j 12} r s^{3}
\end{gathered}
$$

The integrals of the shape functions in local co - ordinates are as follows:

$$
\begin{aligned}
& q_{1}=\iint \frac{\partial^{2} N_{i}}{\partial r^{2}} \frac{\partial^{2} N_{j}}{\partial r^{2}} d r d s=16\left[a_{i 4} a_{j 4}+3 a_{i 7} a_{j 7}+\frac{1}{3} a_{i 8} a_{j 8}+a_{i 11} a_{j 11}\right] \\
& q_{2}=\iint \frac{\partial^{2} N_{i}}{\partial s^{2}} \frac{\partial^{2} N_{j}}{\partial s^{2}} d r d s=16\left[a_{i 6} a_{j 6}+\frac{1}{3} a_{i 9} a_{j 9}+3 a_{i 10} a_{j 10}+a_{i 12} a_{j 12}\right] \\
& q_{3}=\iint \frac{\partial^{2} N_{i}}{\partial r^{2}} \frac{\partial^{2} N_{j}}{\partial s^{2}} d r d s=16\left[a_{i 4} a_{j 6}+a_{i 7} a_{j 9}+a_{i 8} a_{j 10}+a_{i 11} a_{j 12}\right] \\
& q_{4}=\iint \frac{\partial^{2} N_{i}}{\partial s^{2}} \frac{\partial^{2} N_{j}}{\partial r^{2}} d r d s=16\left[a_{i 6} a_{j 4}+a_{i 9} a_{j 7}+a_{i 10} a_{j 8}+a_{i 12} a_{j 11}\right] \\
& q_{5}=\iint \frac{\partial^{2} N_{i}}{\partial r^{2}} \frac{\partial^{2} N_{j}}{\partial r \partial s} d r d s=8\left[a_{i 4} a_{j 5}+a_{i 4} a_{j 11}+2 a_{i 7} a_{j 8}+a_{i 4} a_{j 12}\right. \\
& \left.+\frac{2}{3} a_{i 8} a_{j 9}\right] \\
& q_{6}=\iint \frac{\partial^{2} N_{i}}{\partial r \partial s} \frac{\partial^{2} N_{j}}{\partial r^{2}} d r d s=8\left[a_{i 5} a_{j 4}+2 a_{i 8} a_{j 7}+a_{i 11} a_{j 4}+\frac{2}{3} a_{i 9} a_{j 8}\right. \\
& \left.+a_{i 12} a_{j 4}\right] \\
& q_{7}=\iint \frac{\partial^{2} N_{i}}{\partial s^{2}} \frac{\partial^{2} N_{j}}{\partial r \partial s} d r d s=8\left[a_{i 6} a_{j 5}+a_{i 6} a_{j 11}+\frac{2}{3} a_{i 9} a_{j 8}\right] \\
& q_{8}=\iint \frac{\partial^{2} N_{i}}{\partial r \partial s} \frac{\partial^{2} N_{j}}{\partial s^{2}} d r d s=8\left[a_{i 5} a_{j 6}+\frac{2}{3} a_{i 8} a_{j 9}+a_{i 11} a_{j 6}\right]
\end{aligned}
$$




$$
\begin{aligned}
& q_{9}=\iint \frac{\partial^{2} N_{i}}{\partial r \partial s} \frac{\partial^{2} N_{j}}{\partial r \partial s} d r d s=4\left[a_{i 5} a_{j 5}+a_{i 5} a_{j 11}+\frac{4}{3} a_{i 8} a_{j 8}+a_{i 5} a_{j 12}\right. \\
& \left.+\frac{4}{3} a_{i 9} a_{j 9}+a_{i 11} a_{j 12}+a_{i 12} a_{j 11}+\frac{9}{5} a_{i 12} a_{j 12}\right] \\
& q_{10}=\iint \frac{\partial N_{i}}{\partial r} \frac{\partial N_{j}}{\partial r} d r d s=4\left[a_{i 2} a_{j 2}+\frac{1}{3}\left(3 a_{i 2} a_{j 7}+4 a_{i 4} a_{j 4}+3 a_{i 7} a_{j 2}\right.\right. \\
& +a_{i 7} a_{j 9}+a_{i 5} a_{j 5}+a_{i 9} a_{j 2}+a_{i 5} a_{j 11}+a_{i 7} a_{j 9}+\frac{4}{3} a_{i 8} a_{j 8}+a_{i 9} a_{j 7} \\
& \left.a_{i 11} a_{j 5}\right)+\frac{1}{5}\left(a_{i 5} a_{j 12}+a_{i 9} a_{j 9}+a_{i 12} a_{j 5}+9 a_{i 7} a_{j 7}+3 a_{i 11} a_{j 11}+a_{i 11} a_{j 12}\right. \\
& \left.\left.+a_{i 12} a_{j 11}\right)+\frac{1}{7} a_{i 12} a_{j 12}\right] \\
& q_{11}=\iint \frac{\partial N_{i}}{\partial s} \frac{\partial N_{j}}{\partial s} d r d s=4\left[a_{i 3} a_{j 3}+\frac{1}{3}\left(a_{i 3} a_{j 8}+a_{i 5} a_{j 5}+a_{i 8} a_{j 3}\right.\right. \\
& +3 a_{i 3} a_{j 10}+4 a_{i 6} a_{j 6}+3 a_{i 10} a_{j 3}+a_{i 5} a_{j 12}+a_{i 8} a_{j 10}+\frac{4}{3} a_{i 9} a_{j 9}+a_{i 10} a_{j 8} \\
& \left.+a_{i 12} a_{j 5}\right)+\frac{1}{5}\left(a_{i 5} a_{j 11}+a_{i 8} a_{j 8}+a_{i 11} a_{j 5}+9 a_{i 10} a_{j 10}+a_{i 11} a_{j 12}+a_{i 12} a_{j 11}\right. \\
& \left.\left.+3 a_{i 2} a_{j 12}\right)+\frac{1}{7} a_{i 11} a_{j 11}\right] \\
& q_{12}=\iint \frac{\partial N_{i}}{\partial r} \frac{\partial N_{j}}{\partial s} d r d s=4\left[a_{i 2} a_{j 3}+\frac{1}{3}\left(a_{i 2} a_{j 8}+2 a_{i 4} a_{j 5}+3 a_{i 7} a_{j 8}\right.\right. \\
& +3 a_{i 2} a_{j 10}+2 a_{i 5} a_{j 6}+a_{i 9} a_{j 3}+2 a_{i 4} a_{j 12}+3 a_{i 7} a_{j 10}+\frac{4}{3} a_{i 8} a_{j 9}+\frac{1}{3} a_{i 9} a_{j 8} \\
& \left.\left.+2 a_{i 11} a_{j 6}\right)\right] \\
& q_{13}=\iint \frac{\partial N_{i}}{\partial s} \frac{\partial N_{j}}{\partial r} d r d s=4\left[a_{i 3} a_{j 2}+\frac{1}{3}\left(3 a_{i 3} a_{j 7}+2 a_{i 5} a_{j 4}+a_{i 8} a_{j 2}\right.\right. \\
& +a_{i 3} a_{j 9}+2 a_{i 6} a_{j 5}+3 a_{i 10} a_{j 2}+2 a_{i 6} a_{j 11}+\frac{1}{3} a_{i 8} a_{j 9}+\frac{4}{3} a_{i 9} a_{j 8}+3 a_{i 10} a_{j 7} \\
& \left.\left.+2 a_{i 12} a_{j 4}\right)+\frac{1}{5}\left(2 a_{i 6} a_{j 12}+3 a_{i 10} a_{j 9}+3 a_{i 8} a_{j 7}+2 a_{i 11} a_{j 4}\right)\right]
\end{aligned}
$$

The values of the integrals are converted from local co - ordinate $(r, s)$ to global co - ordinates.

The integrals of the shape functions in global co - ordinates are as follows:

$$
\begin{aligned}
& r_{1}=\iint \frac{\partial^{2} N_{i}}{\partial x^{2}} \frac{\partial N_{j}}{\partial x^{2}} d x d y=\left(\frac{4 h_{y}}{h_{x}^{3}}\right) q_{1}=\frac{4 n^{3} b}{m a^{3}} q_{1} \\
& r_{2}=\iint \frac{\partial^{2} N_{i}}{\partial y^{2}} \frac{\partial^{2} N_{j}}{\partial y^{2}} d x d y=\left(\frac{4 h_{x}}{h_{y}^{3}}\right) q_{2}=\frac{4 a m^{3}}{n b^{3}} q_{2} \\
& r_{3}=\iint \frac{\partial^{2} N_{i}}{\partial x^{2}} \frac{\partial^{2} N_{j}}{\partial y^{2}} d x d y=\left(\frac{4}{h_{y} h_{x}}\right) q_{3}=\frac{4 m n}{a b} q_{3} \\
& r_{4}=\iint \frac{\partial^{2} N_{i}}{\partial y^{2}} \frac{\partial^{2} N_{j}}{\partial x^{2}} d x d y=\left(\frac{4}{h_{y} h_{x}}\right) q_{4}=\frac{4 m n}{a b} q_{4} \\
& r_{5}=\iint \frac{\partial^{2} N_{i}}{\partial x^{2}} \frac{\partial^{2} N_{j}}{\partial x \partial y} d x d y=\left(\frac{4}{h_{x}^{2}}\right) q_{5}=\frac{4 n^{2}}{a^{2}} q_{5} \\
& r_{6}=\iint \frac{\partial^{2} N_{i}}{\partial x \partial y} \frac{\partial^{2} N_{j}}{\partial x^{2}} d x d y=\left(\frac{4}{h_{x}^{2}}\right) q_{6}=\frac{4 n^{2}}{a^{2}} q_{6}
\end{aligned}
$$

Suleiman, O. M. E., Osman, M. Y., \& Hassan, T. (2019). Stability of thin laminated decks plates under plane compressive loading. International Research Journal of Engineering, IT \& Scientific Research, 5(2), 1-28. 
$r_{7}=\iint \frac{\partial^{2} N_{i}}{\partial y^{2}} \frac{\partial^{2} N_{j}}{\partial x \partial y} d x d y=\left(\frac{4}{h_{y}^{2}}\right) q_{7}=\frac{4 m^{2}}{a^{2}} q_{7}$

$r_{8}=\iint \frac{\partial^{2} N_{i}}{\partial x \partial y} \frac{\partial^{2} N_{j}}{\partial y^{2}} d x d y=\left(\frac{4}{h_{y}^{2}}\right) q_{8}=\frac{4 m^{2}}{b^{2}} q_{8}$

$r_{9}=\iint \frac{\partial^{2} N_{i}}{\partial x \partial y} \frac{\partial^{2} N_{j}}{\partial x \partial y} d x d y=\left(\frac{4}{h_{y} h_{x}}\right) q_{9}=\frac{4 m n}{a b} q_{9}$

$r_{10}=\iint \frac{\partial N_{i}}{\partial x} \frac{\partial N_{j}}{\partial x} d x d y=\left(\frac{h_{y}}{h_{x}}\right) q_{10}=\frac{b n}{a m} q_{10}$

$r_{11}=\iint \frac{\partial N_{i}}{\partial y} \frac{\partial N_{j}}{\partial y} d x d y=\left(\frac{h_{x}}{h_{y}}\right) q_{11}=\frac{a m}{b n} q_{11}$

$r_{12}=\iint \frac{\partial N_{i}}{\partial x} \frac{\partial N_{j}}{\partial y} d x d y=q_{12}$

$r_{13}=\iint \frac{\partial N_{i}}{\partial y} \frac{\partial N_{j}}{\partial x} d x d y=q_{13}$

In the previous equations $h_{x}=\frac{a}{n}$ and $h_{y}=\frac{b}{m}$ where $a$ and $b$ are the lengths of the plate along the $x-$ and $y-$ axis respectively. $n$ and $m$ are the number of elements in the $x$ - and $y$-directions respectively.

The elements of the stiffness matrix and the differential matrix can be written as follows:

$K_{i j}=D_{11} r_{1}+D_{12} r_{4}+2 D_{16} r_{3}+D_{12} r_{3}+D_{22} r_{2}+2 D_{66} r_{8}+2 D_{16} r_{5}+2 D_{26} r_{7}+4 D_{66} r_{9}$

$K_{i j}^{D}=P_{x} r_{10}+P_{x y}\left(r_{12}+r_{13}\right)+P_{y} r_{11}$

or in the non - dimensional form:

$K_{i j}=\frac{4 n^{3}}{m}\left(\frac{b}{a}\right) \bar{D}_{11} q_{1}+4 m n\left(\frac{a}{b}\right) \bar{D}_{12} q_{4}+4 n^{2} \bar{D}_{16} q_{6}+4 m n\left(\frac{a}{b}\right) \bar{D}_{12} q_{3}$

$+\frac{4 m^{3}}{n}\left(\frac{a}{b}\right) \bar{D}_{22} q_{2}+4 m^{2}\left(\frac{a}{b}\right)^{2} \bar{D}_{26} q_{8}+4 n^{2} \bar{D}_{16} q_{5}+4 m^{2}\left(\frac{a}{b}\right)^{2} \bar{D}_{26} q_{7}$

$+4 m n\left(\frac{a}{b}\right) \bar{D}_{66} q_{9}$

$K_{i j}^{D}=\bar{P}_{x} \frac{n}{m}\left(\frac{b}{a}\right) q_{10}+\bar{P}_{x y}\left(q_{12}+q_{13}\right)+\bar{P}_{y} \frac{m}{n}\left(\frac{a}{b}\right) q_{11}$

where

$$
\bar{D}_{i j}=\left(\frac{1}{E_{1} h^{3}}\right) D_{i j}, \quad \bar{P}_{i}=\left(\frac{a}{E_{1} h^{3}}\right) P_{i}
$$

Also

$$
\bar{w}=\left(\frac{1}{h}\right) w, \quad \bar{\phi}=\left(\frac{h}{a}\right) \phi, \quad \bar{\psi}=\left(\frac{h}{a}\right) \psi, \quad \bar{b}=b / a
$$

The transformed stiffnesses are as follows:

$C_{11}=C_{11}^{\prime} c^{4}+2 c^{2} s^{2}\left(C_{11}^{\prime}+2 C_{66}^{\prime}\right)+C_{22}^{\prime} s^{4}$

$C_{12}=c^{2} s^{2}\left(C_{11}^{\prime}+C_{22}^{\prime}+4 C_{66}^{\prime}\right)+C_{12}^{\prime}\left(c^{4}+s^{4}\right)$

$C_{16}=c s\left[C_{11}^{\prime} c^{4}+C_{22}^{\prime} s^{2}-\left(C_{12}^{\prime}+2 C_{66}^{\prime}\right)\left(c^{2}-s^{2}\right)\right]$

$C_{22}=C_{11}^{\prime} s^{4}+2 c^{2} s^{2}\left(C_{12}^{\prime}+2 C_{66}^{\prime}\right)+C_{22}^{\prime} c^{4}$ 


$$
\begin{aligned}
& C_{26}=c s\left[C_{11}^{\prime} s^{2}-C_{22}^{\prime} c^{2}-\left(C_{12}^{\prime}+2 C_{66}^{\prime}\right)\left(c^{2}-s^{2}\right)\right] \\
& C_{66}=\left(C_{11}^{\prime}+C_{22}^{\prime}-2 C_{12}^{\prime}\right) c^{2} s^{2}+C_{66}^{\prime}\left(c^{2}-s^{2}\right)^{2}
\end{aligned}
$$

Where

$C_{11}^{\prime}=\frac{E_{1}}{1-v_{12} v_{21}}$

$C_{12}^{\prime}=\frac{v_{21} E_{1}}{1-v_{12} v_{21}}=\frac{v_{12} E_{1}}{1-v_{12} v_{21}}$

$C_{22}^{\prime}=\frac{E_{2}}{1-v_{12} v_{21}}$

$C_{44}^{\prime}=G_{23}, \quad C_{55}^{\prime}=G_{13}$ and $C_{66}^{\prime}=G_{12}$

$E_{1}$ and $E_{2}$ are the elastic moduli in the direction of the fiber and the transverse directions respectively, $v$ is the Poisson's ratio. $G_{12}, G_{13}$, and $G_{23}$ are the shear moduli in the $x-y$ plane, $y-z$ plane, and $x-z$ plane respectively, and the subscripts 1 and 2 refer to the direction of fiber and the transverse direction respectively

\section{Results and Discussions}

\section{Numerical Results}

\subsection{Effect of Lamination Scheme}

In the present analysis the lamination scheme of plates is supposed to be symmetric, anti - symmetric and quasi isotropic.

Four lamination schemes were considered which are symmetric and anti - symmetric cross - ply and angle - ply laminates. Table 1 gives a comparison between the non - dimensional buckling loads for all lamination schemes. The results are shown graphically in Figure 6. The thickness of all layers is assumed equal, the length to thickness ratio $(a / h=20)$, and the modulus ratio $\left(E_{1} / E_{2}=5\right)$. It is noticed from Table 1 and Figures 6, 7 and 8 that the values of the non - dimensional buckling loads for both symmetric and anti - symmetric lamination are slightly different, except for symmetric and anti - symmetric angle - ply laminates which are exactly the same. Because of this fact, the rest of the upcoming effects will be discussed for symmetric case only. The results indicate that the symmetric laminate is stiffer than the anti - symmetric one. This phenomenon is caused by coupling between bending and stretching which lowers the buckling loads of symmetric laminate.

Table 1

The first five non - dimensional buckling loads $\overline{\mathrm{P}}=\mathrm{Pa}^{2} / \mathrm{E}_{1} \mathrm{~h}^{3}$ of symmetric cross $-\mathrm{ply}(0 / 90 / 90 / 0)$ and anti symmetric cross - ply $(0 / 90 / 0 / 90)$, and symmetric angle - ply $(45 /-45 /-45 / 45)$ and anti - symmetric angle - ply (45/-45/45/-45) laminated plates with $a / h=20$, and $E_{1} / E_{2}=5$

\begin{tabular}{ccccc}
\hline Lamination & Mode & \multicolumn{3}{c}{ Boundary Conditions } \\
\cline { 3 - 5 } Scheme & Number & SS & CC & CS \\
\hline & 1 & 0.6972 & 2.1994 & 1.8225 \\
$0 / 90 / 90 / 0$ & 2 & 1.2522 & 2.5842 & 2.0097 \\
& 3 & 2.4284 & 4.1609 & 2.7116 \\
& 4 & 2.6907 & 4.7431 & 4.3034 \\
& 5 & 2.7346 & 5.0168 & 4.4536 \\
$0 / 90 / 0 / 90$ & 1 & 0.6973 & 2.2273 & 1.5591 \\
& 2 & 1.9947 & 3.9687 & 2.3391 \\
& 3 & 1.9958 & 3.9732 & 3.7581 \\
& 4 & 2.6912 & 4.7871 & 3.8290 \\
& 5 & 4.3962 & 7.0544 & 4.5402 \\
\hline
\end{tabular}

Suleiman, O. M. E., Osman, M. Y., \& Hassan, T. (2019). Stability of thin laminated decks plates under plane compressive loading. International Research Journal of Engineering, IT \& Scientific Research, 5(2), 1-28. 


\begin{tabular}{lllll}
\hline & 1 & 0.8729 & 1.9505 & 1.4756 \\
$45 /-45 /-45 / 45$ & 2 & 1.6400 & 2.8534 & 2.1162 \\
& 3 & 2.3130 & 3.8941 & 3.3039 \\
& 4 & 2.7100 & 4.3753 & 3.3068 \\
& 5 & 3.5488 & 5.2694 & 4.4166 \\
$45 /-45 / 45 /-45$ & 1 & 0.8729 & 2.2010 & 1.6554 \\
& 2 & 1.6400 & 3.7616 & 2.5672 \\
& 3 & 2.3130 & 3.7654 & 3.4642 \\
& 4 & 2.7100 & 5.6599 & 4.2174 \\
& 5 & 3.5488 & 5.9540 & 4.8091 \\
\hline
\end{tabular}

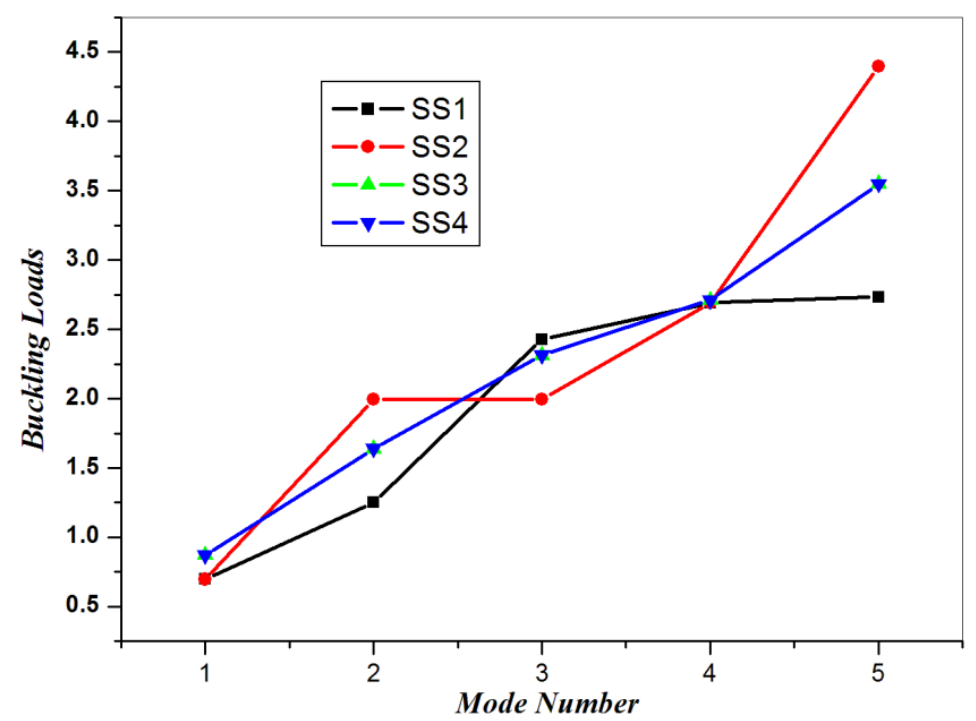

Figure 6. Effect of lamination scheme for simply supported laminates

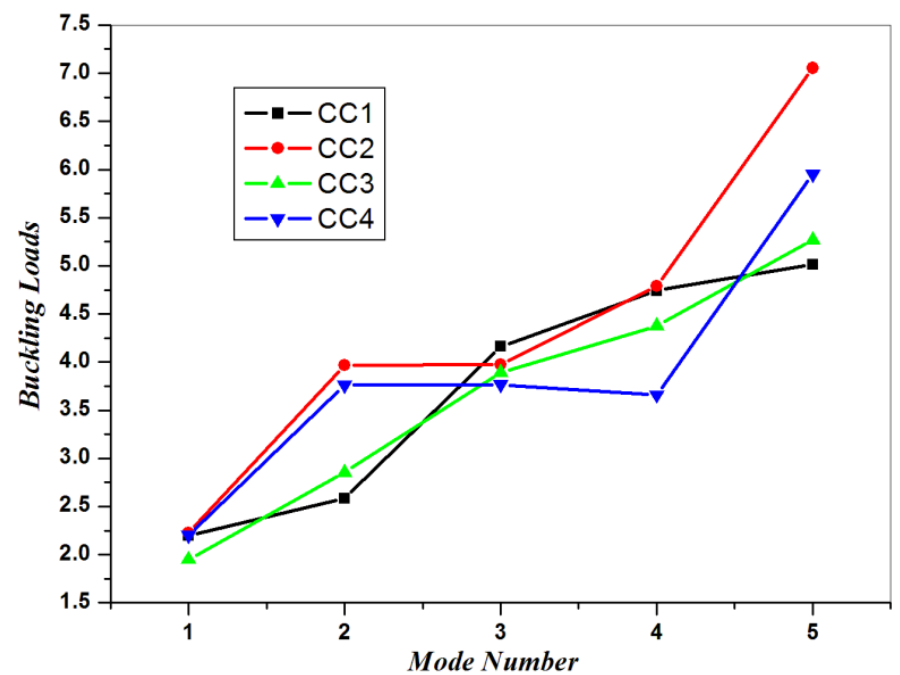

Figure 7. Effect of lamination scheme for clamped - clamped laminates 


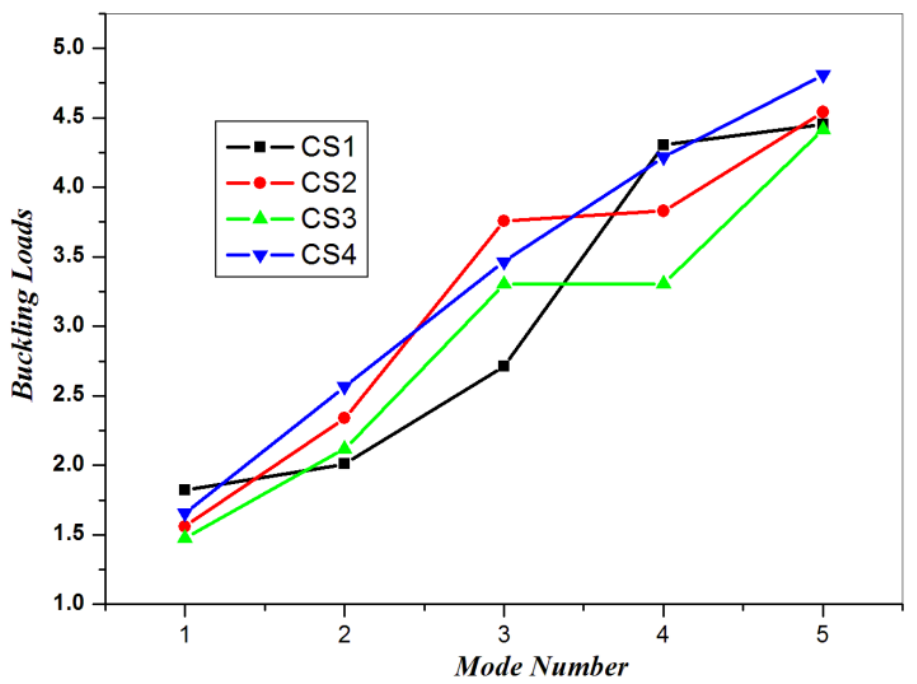

Figure 8. Effect of lamination scheme for clamped - simply supported laminates

Tables 2 and 3 show the buckling load of quasi - isotropic rectangular composite plate with $a / h=20, a / b=1$ and different modulus ratios $\left(E_{1} / E_{2}=40\right.$ and 5$)$. The buckling load is highly influenced by its boundary conditions. The buckling load of the quasi - isotropic $(0 /+45 /-45 / 90)$ rectangular composite plate with CC type boundary condition is 1.5 times higher than the buckling load of the composite plate with CS type boundary condition and more than 3 times of SS type boundary condition.

Table 2

The first three non - dimensional buckling loads of quasi - isotropic (0/+45/-45/90) laminated plates with a/h=20, and $E_{1} / E_{2}=40$

\begin{tabular}{cccc}
\hline Mode & \multicolumn{3}{c}{ Boundary Conditions } \\
\cline { 2 - 4 } Number & SS & CC & CS \\
\hline 1 & 0.4905 & 1.6878 & 1.1683 \\
2 & 1.4842 & 3.0187 & 1.7359 \\
3 & 1.4850 & 3.0229 & 2.7673 \\
\hline
\end{tabular}

Table 3

The first three non - dimensional buckling load of quasi - isotropic $(0 /+45 /-45 / 90)$ laminated plates with a/h=20, and

$$
\mathrm{E}_{1} / \mathrm{E}_{2}=5
$$

\begin{tabular}{cccc}
\hline Mode & \multicolumn{3}{c}{ Boundary Conditions } \\
\cline { 2 - 4 } Number & SS & CC & CS \\
\hline 1 & 0.7338 & 2.2255 & 1.5717 \\
2 & 2.0202 & 3.9506 & 2.3714 \\
3 & 2.0214 & 3.9549 & 3.7214 \\
\hline
\end{tabular}

Suleiman, O. M. E., Osman, M. Y., \& Hassan, T. (2019). Stability of thin laminated decks plates under plane compressive loading. International Research Journal of Engineering, IT \& Scientific Research, 5(2), 1-28. 


\subsection{Effect of Aspect Ratio}

In this study, the buckling loads for symmetrically loaded laminated composite plates of layer orientation 0/90/90/0 have been determined for seven different aspect ratios ranging from 0.5 to 2.0 and two modulus ratios 40 and 5 as shown in Tables 4 and 5 and Figures 9 and 10. The first mode of buckling loads was considered. It is observed that the buckling load increases continuously with increasing aspect ratio but the rate of increase is not uniform. This may be due to the effect of bending - extensional twisting stiffness which increases the critical load. The buckling load is maximum for clamped - clamped (CC), clamped - simply supported (CS) while minimum for simply - simply supported (SS) boundary conditions. This means that as the plate becomes more restrained, its resistance to buckling increases. The reason is that the structural stiffness reduces due to its constrains.

Table 4

The first three non - dimensional buckling loads $\overline{\mathrm{P}}=\mathrm{Pa}^{2} / \mathrm{E}_{1} \mathrm{~h}^{3}$ of symmetric cross - ply $(0 / 90 / 90 / 0)$ laminated plates with $\mathrm{a} / \mathrm{h}=20$, and $\mathrm{E}_{1} / \mathrm{E}_{2}=40$

\begin{tabular}{ccccc}
\hline $\begin{array}{c}\text { Aspect Ratio } \\
(a / b)\end{array}$ & $\begin{array}{c}\text { Mode } \\
\text { Number }\end{array}$ & SS & CC & CS \\
\hline & 1 & 0.4143 & 1.0742 & 0.9679 \\
0.5 & 2 & 0.4236 & 1.0941 & 1.0484 \\
& 3 & 0.5408 & 1.3751 & 1.1257 \\
& 1 & 0.4300 & 1.2389 & 1.0444 \\
0.75 & 2 & 0.4978 & 1.2691 & 1.2043 \\
& 3 & 0.6520 & 1.8354 & 1.2921 \\
& 1 & 0.4409 & 1.3795 & 1.0723 \\
1.0 & 2 & 0.5580 & 1.5286 & 1.3105 \\
& 3 & 1.0763 & 2.1648 & 1.6946 \\
& 1 & 0.4224 & 1.5549 & 1.1349 \\
1.25 & 2 & 0.7795 & 1.7455 & 1.4327 \\
& 3 & 1.6164 & 3.0019 & 1.8042 \\
& 1 & 0.4400 & 1.6402 & 1.2543 \\
1.5 & 2 & 1.0787 & 2.2999 & 1.3330 \\
& 3 & 1.6841 & 3.2702 & 2.4753 \\
& 1 & 0.4885 & 1.8361 & 1.1494 \\
1.75 & 2 & 1.4473 & 3.0138 & 1.6342 \\
& 3 & 1.8520 & 3.6574 & 2.7310 \\
& 1 & 0.5642 & 2.1358 & 1.1054 \\
2.0 & 2 & 1.7525 & 3.7696 & 2.0207 \\
& 3 & 1.8813 & 3.8703 & 2.8553 \\
\hline
\end{tabular}

Table 5

The first three non - dimensional buckling loads $\overline{\mathrm{P}}=\mathrm{Pa}^{2} / \mathrm{E}_{1} \mathrm{~h}^{3}$ of symmetric cross - ply $(0 / 90 / 90 / 0)$ laminated plates with $\mathrm{a} / \mathrm{h}=20$, and $\mathrm{E}_{1} / \mathrm{E}_{2}=5$

\begin{tabular}{ccccc}
\hline $\begin{array}{c}\text { Aspect } \\
\text { Ratio } \\
(a / b)\end{array}$ & $\begin{array}{c}\text { Mode } \\
\text { Number }\end{array}$ & \multicolumn{4}{c}{ SS } & CC & CS \\
\hline \multirow{3}{*}{0.5} & 1 & 0.6787 & 1.7786 & 1.6325 \\
& 2 & 0.6841 & 1.8364 & 1.7192 \\
& 3 & 0.8672 & 2.2141 & 1.9284 \\
0.75 & 1 & 0.6698 & 2.0107 & 1.7117 \\
& 2 & 0.8831 & 2.1504 & 1.9339 \\
& 3 & 1.4912 & 2.7694 & 2.2689 \\
1.0 & 1 & 0.6972 & 2.1994 & 1.8225 \\
& 2 & 1.2552 & 2.5842 & 2.0097 \\
\hline
\end{tabular}




\begin{tabular}{lllll}
\hline & 3 & 2.4284 & 4.1609 & 2.7116 \\
\multirow{4}{*}{1.25} & 1 & 0.7726 & 2.3958 & 1.8397 \\
& 2 & 1.7753 & 3.5341 & 2.1821 \\
& 3 & 2.6844 & 5.1641 & 3.8539 \\
1.5 & 1 & 0.8943 & 2.7961 & 1.7643 \\
& 2 & 2.4305 & 4.8034 & 2.7358 \\
& 3 & 2.6675 & 5.2420 & 4.6305 \\
1.75 & 1 & 1.0588 & 3.3873 & 1.7741 \\
& 2 & 2.6919 & 5.4542 & 3.4532 \\
& 3 & 3.2171 & 6.3629 & 4.7373 \\
2.0 & 1 & 1.2630 & 4.1517 & 1.8578 \\
& 2 & 2.7619 & 5.8342 & 4.3179 \\
& 3 & 4.1301 & 8.1942 & 4.6131 \\
\hline
\end{tabular}

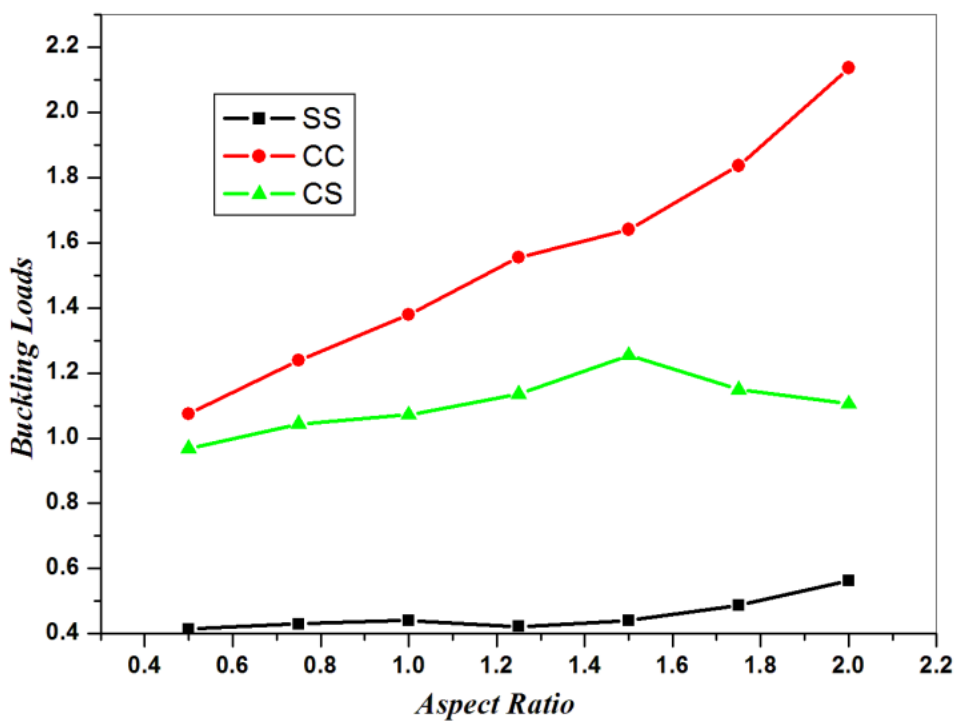

Figure 9. Effect of aspect ratio for different boundary conditions,

$$
E_{1} / E_{2}=40
$$

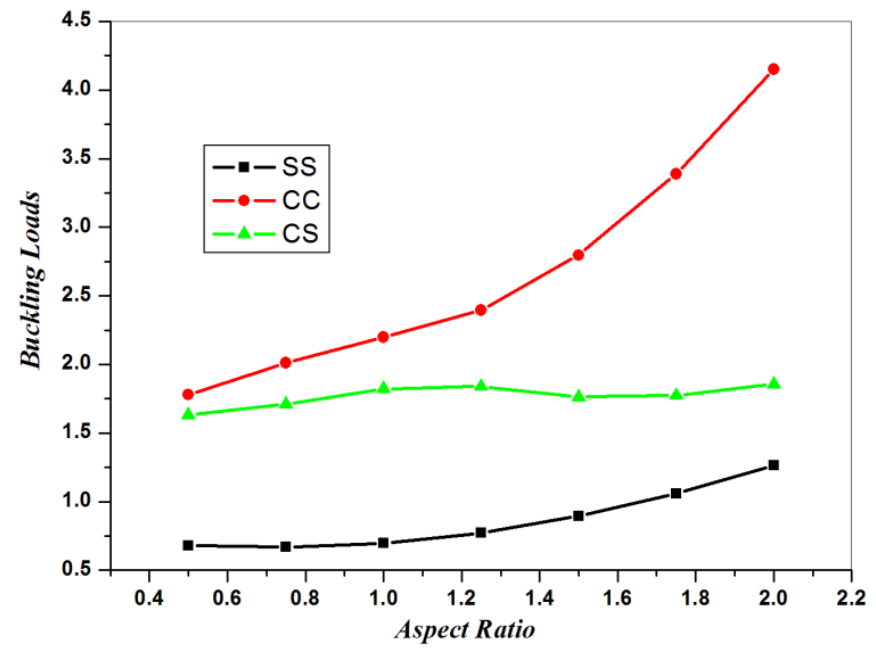

Figure 10. Effect of aspect ratio for different boundary conditions,

$$
E_{1} / E_{2}=5
$$

Suleiman, O. M. E., Osman, M. Y., \& Hassan, T. (2019). Stability of thin laminated decks plates under plane compressive loading. International Research Journal of Engineering, IT \& Scientific Research, 5(2), 1-28. 


\subsection{Effect of Material Anisotropy}

The buckling loads as a function of modulus ratio of symmetric cross - ply plates (0/90/90/ 0) are illustrated in Table 6 and Figure 11. As confirmed by other investigators, the buckling load decreases with increase in modulus ratio. Therefore, the coupling effect on buckling loads is more pronounced with the increasing degree of anisotropy. It is observed that the variation of buckling load becomes almost constant for higher values of elastic modulus ratio.

Table 6

The first three non - dimensional buckling loads $\overline{\mathrm{P}}=\mathrm{Pa}^{2} / \mathrm{E}_{1} \mathrm{~h}^{3}$ of symmetric cross - ply $(0 / 90 / 90 / 0)$ square laminated plates for different modulus ratios with $\mathrm{a} / \mathrm{h}=20$

\begin{tabular}{ccccc}
\hline \multirow{2}{*}{$E_{1} / E_{2}$} & Mode & \multicolumn{3}{c}{ Boundary Conditions } \\
\cline { 3 - 5 } Number & SS & CC & CS \\
\hline \multirow{3}{*}{5} & 1 & 0.6972 & 2.1994 & 1.8225 \\
& 2 & 1.2552 & 2.5842 & 2.0097 \\
& 3 & 2.4284 & 4.1609 & 2.7116 \\
\multirow{3}{*}{10} & 1 & 0.5505 & 1.8548 & 1.3928 \\
& 2 & 0.8557 & 1.8951 & 1.8292 \\
& 3 & 1.6532 & 2.9814 & 1.9089 \\
15 & 1 & 0.5019 & 1.6663 & 1.2505 \\
& 2 & 0.7232 & 1.7248 & 1.6428 \\
& 3 & 1.3966 & 2.6049 & 1.7694 \\
20 & 1 & 0.4775 & 1.5515 & 1.1791 \\
& 2 & 0.6569 & 1.6524 & 1.5096 \\
& 3 & 1.2683 & 2.4228 & 1.7394 \\
25 & 1 & 0.4629 & 1.4828 & 1.1365 \\
& 2 & 0.6172 & 1.6055 & 1.4299 \\
& 3 & 1.1916 & 2.3171 & 1.7214 \\
30 & 1 & 0.4531 & 1.4366 & 1.1078 \\
& 2 & 0.5907 & 1.5723 & 1.3766 \\
& 3 & 1.1402 & 2.2481 & 1.7094 \\
35 & 1 & 0.4462 & 1.4044 & 1.0877 \\
& 2 & 0.5723 & 1.5479 & 1.3391 \\
& 3 & 1.1043 & 2.2006 & 1.7009 \\
40 & 1 & 0.4409 & 1.3795 & 1.0723 \\
& 2 & 0.5580 & 1.5286 & 1.3105 \\
& 3 & 1.0763 & 2.1648 & 1.6946 \\
\hline \multirow{3}{*}{3}
\end{tabular}




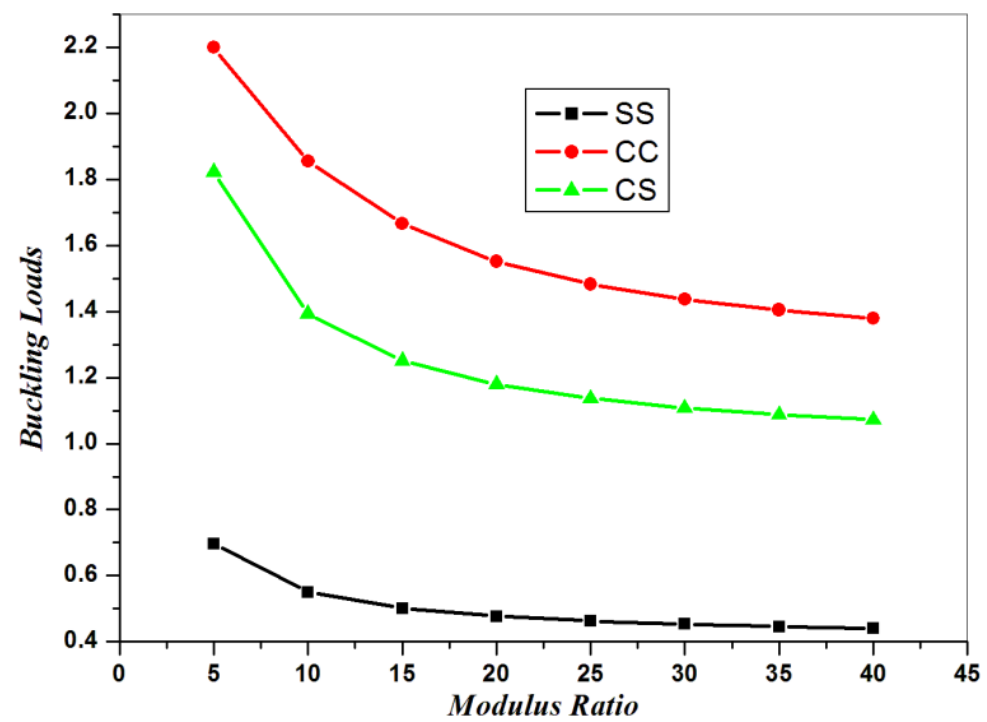

Figure 11. Effect of material anisotropy

\subsection{Effect of Fiber Orientations of Layers}

The variation of the buckling load, $\overline{\boldsymbol{P}}$ with fiber orientation $(\boldsymbol{\theta})$ of square laminated plate is shown in Tables 7 and 8, and Figs. 12 and 13. Three boundary conditions SS, CC and CS are considered in this case. The buckling loads have been determined for two modulus ratios 40 and 5. The curves of simply - simply supported (SS) boundary conditions show maximum value of buckling load at $=\mathbf{4 5}^{\boldsymbol{o}}$. However, this trend is different for a plate under clamped - clamed (CC) boundary conditions which show minimum buckling load at $\boldsymbol{\theta}=\mathbf{4 5}^{\boldsymbol{o}}$. For clamped simply supported, it is observed that the buckling load decreases continuously with $\boldsymbol{\theta}$, this may be due to the total and partial fixed rotation ( $\boldsymbol{\phi}$ and $\boldsymbol{\psi})$ in the two later cases.

Table 7

The first three non - dimensional buckling loads $\overline{\mathrm{P}}=\mathrm{Pa}^{2} / \mathrm{E}_{1} \mathrm{~h}^{3}$ of laminated plates for different fiber orientations ( $\theta$ ) with $\mathrm{a} / \mathrm{h}=20$, and $\mathrm{E}_{1} / \mathrm{E}_{2}=40$

\begin{tabular}{ccccc}
\hline Orientation & Mode & \multicolumn{3}{c}{ Boundary Conditions } \\
Angle $(\theta)$ & Number & SS & CC & CS \\
\hline \multirow{2}{*}{0} & 1 & 0.2604 & 0.6134 & 0.5561 \\
& 2 & 0.2825 & 0.6398 & 0.5729 \\
& 3 & 0.3960 & 0.8738 & 0.6745 \\
15 & 1 & 0.2759 & 0.5957 & 0.5496 \\
& 2 & 0.3171 & 0.6123 & 0.5855 \\
& 3 & 0.4771 & 0.8638 & 0.7570 \\
30 & 1 & 0.2823 & 0.5636 & 0.5114 \\
& 2 & 0.3125 & 0.5834 & 0.5352 \\
45 & 3 & 0.4861 & 0.9552 & 0.7902 \\
& 1 & 0.2773 & 0.5207 & 0.4230 \\
& 2 & 0.3253 & 0.5842 & 0.4490 \\
60 & 3 & 0.5135 & 0.9793 & 0.7093 \\
& 1 & 0.2834 & 0.5574 & 0.3073 \\
& 2 & 0.3116 & 0.5788 & 0.3895 \\
75 & 3 & 0.4783 & 0.9107 & 0.6362 \\
& 1 & 0.2762 & 0.5859 & 0.3137 \\
& 2 & 0.3153 & 0.6043 & 0.3297 \\
\hline
\end{tabular}

Suleiman, O. M. E., Osman, M. Y., \& Hassan, T. (2019). Stability of thin laminated decks plates under plane compressive loading. International Research Journal of Engineering, IT \& Scientific Research, 5(2), 1-28. 


\begin{tabular}{ccccc}
\hline \multirow{4}{*}{90} & 3 & 0.4161 & 0.8252 & 0.4924 \\
& 1 & 0.2602 & 0.6061 & 0.3069 \\
& 2 & 0.2811 & 0.6260 & 0.3438 \\
& 3 & 0.3908 & 0.8429 & 0.4801 \\
\hline
\end{tabular}

Table 8

The first three non - dimensional buckling loads $\overline{\mathrm{P}}=\mathrm{Pa}^{2} / \mathrm{E}_{1} \mathrm{~h}^{3}$ of laminated plates for different fiber orientations $(\theta)$ with $\mathrm{a} / \mathrm{h}=20$, and $\mathrm{E}_{1} / \mathrm{E}_{2}=5$

\begin{tabular}{ccccc}
\hline Orientation & Mode & \multicolumn{3}{c}{ Boundary Conditions } \\
\cline { 3 - 5 } Angle $(\theta)$ & Number & SS & CC & CS \\
\hline \multirow{2}{*}{0} & 1 & 0.6970 & 2.1130 & 1.6496 \\
& 2 & 1.0086 & 2.1396 & 2.0991 \\
& 3 & 1.7709 & 3.1397 & 2.1597 \\
\hline \multirow{2}{*}{15} & 1 & 0.7108 & 2.0261 & 1.6665 \\
& 2 & 1.0908 & 2.1400 & 1.9833 \\
& 3 & 1.8704 & 3.2340 & 2.2141 \\
\hline \multirow{3}{*}{30} & 1 & 0.7457 & 1.8142 & 1.6326 \\
& 2 & 1.2613 & 2.2494 & 1.7099 \\
& 3 & 2.0671 & 3.4809 & 2.4700 \\
\hline \multirow{2}{*}{45} & 1 & 0.7665 & 1.7189 & 1.3114 \\
& 2 & 1.3477 & 2.3567 & 1.7689 \\
& 3 & 2.1557 & 3.5899 & 2.7032 \\
\hline \multirow{2}{*}{60} & 1 & 0.7457 & 1.8147 & 1.0893 \\
& 2 & 1.2602 & 2.2457 & 1.7913 \\
& 3 & 2.0637 & 3.4650 & 2.6452 \\
\hline \multirow{2}{*}{75} & 1 & 0.7110 & 2.0264 & 0.9824 \\
& 2 & 1.0898 & 2.1366 & 1.6562 \\
& 3 & 1.8659 & 3.2178 & 2.7338 \\
\hline \multirow{2}{*}{90} & 1 & 0.6970 & 2.1101 & 0.9573 \\
& 2 & 1.0080 & 2.1389 & 1.5827 \\
& 3 & 1.7666 & 3.1269 & 2.7322 \\
\hline
\end{tabular}

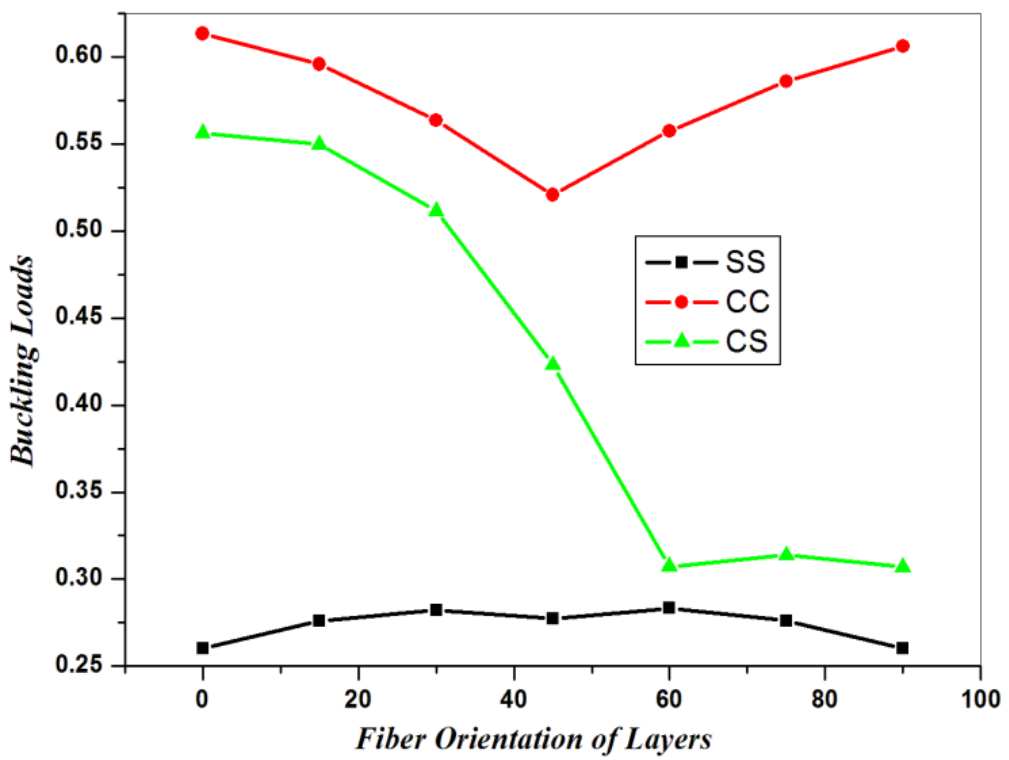

Figure 12. Effect of fiber orientation of layers, $E_{1} / E_{2}=40$ 


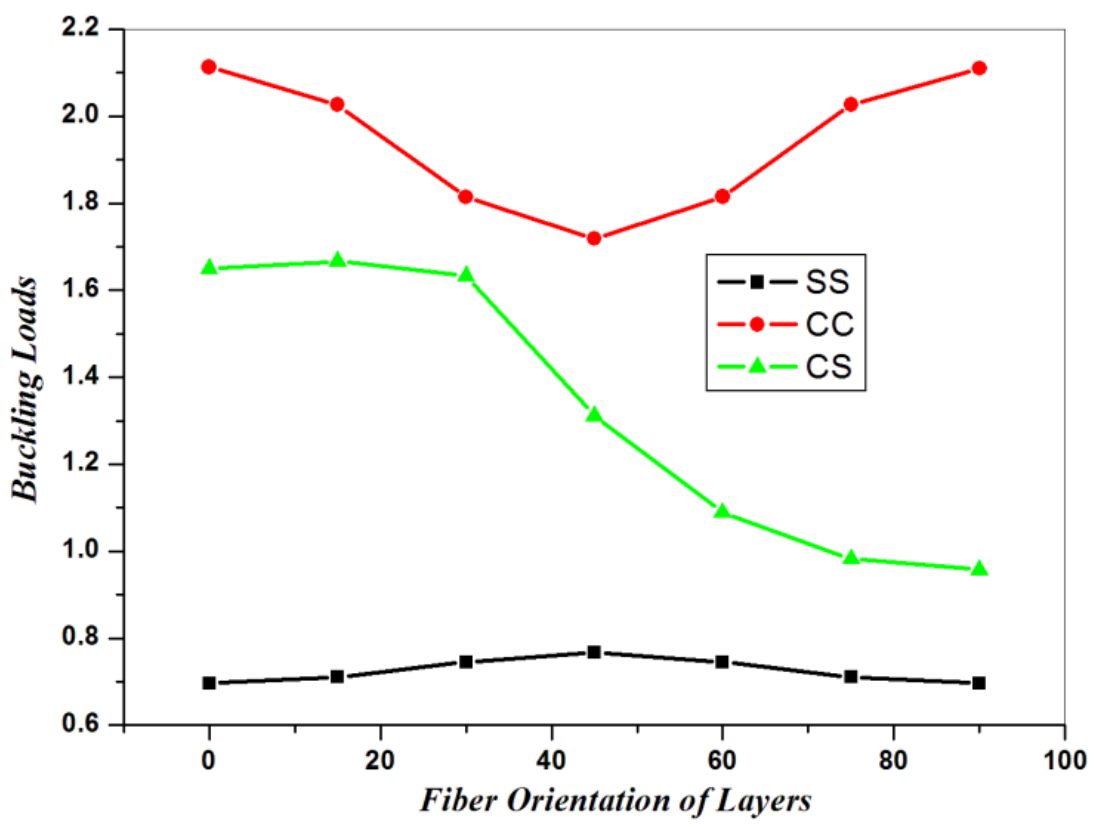

Figure 13. Effect of fiber orientation of layers, $E_{1} / E_{2}=5$

\subsection{Effect of Reversing Lamination Scheme}

In order to study the stacking sequence of laminated plates, two lamination schemes of cross - ply (0/90) and (90/ 0) and two other lamination of angle ply (45/ -45) and (-45/45) were considered. The results of their buckling loads of parameter $\left(\overline{\boldsymbol{P}}=\boldsymbol{P} \boldsymbol{a}^{\mathbf{2}} / \boldsymbol{E}_{\mathbf{1}} \boldsymbol{h}^{\mathbf{3}}\right)$ are given in Tables 9, 10, 11 and 12. Three boundary conditions SS, CC and CS are considered in this case. The buckling loads have been determined for two modulus ratios 40 and 5 . It is observed that, the buckling loads are completely the same for the given first three modes.

Therefore, it can be concluded that the buckling load of laminated plates will remain the same even if the lamination order is reversed. The reason behind this is that the transformed elastic coefficients, $\left[C_{i j}\right]$, are equal for both lamination schemes.

Table 9

Non-dimensional buckling loads $\overline{\mathrm{P}}=\mathrm{Pa}^{2} / \mathrm{E}_{1} \mathrm{~h}^{3}$ of $(0 / 90)$ and (90/0) lamination schemes of square laminated plates with $\mathrm{a} / \mathrm{h}=20$, and $\mathrm{E}_{1} / \mathrm{E}_{2}=40$

\begin{tabular}{ccccc}
\hline \multirow{2}{*}{$\begin{array}{c}\text { Lamination } \\
\text { order }\end{array}$} & Mode & \multicolumn{3}{c}{ Boundary Conditions } \\
\cline { 3 - 5 } & Number & SS & CC & CS \\
\hline \multirow{2}{*}{$0 / 90$} & 1 & 0.4410 & 1.6885 & 1.1512 \\
& 2 & 0.4494 & 3.0311 & 1.6881 \\
& 3 & 1.4502 & 3.0349 & 2.5982 \\
$90 / 0$ & 1 & 0.4410 & 1.6885 & 1.1512 \\
& 2 & 0.4494 & 3.0311 & 1.6881 \\
& 3 & 1.4502 & 3.0349 & 2.5982 \\
\hline
\end{tabular}

Suleiman, O. M. E., Osman, M. Y., \& Hassan, T. (2019). Stability of thin laminated decks plates under plane compressive loading. International Research Journal of Engineering, IT \& Scientific Research, 5(2), 1-28. 
Table 10

Non - dimensional buckling loads $\overline{\mathrm{P}}=\mathrm{Pa}^{2} / \mathrm{E}_{1} \mathrm{~h}^{3}$ of $(0 / 90)$ and $(90 / 0)$ lamination schemes of square laminated plates with $\mathrm{a} / \mathrm{h}=20$, and $\mathrm{E}_{1} / \mathrm{E}_{2}=5$

\begin{tabular}{ccccc}
\hline $\begin{array}{c}\text { Lamination } \\
\text { order }\end{array}$ & Mode & \multicolumn{3}{c}{ Boundary Conditions } \\
Number & SS & CC & CS \\
\hline \multirow{2}{*}{$0 / 90$} & 1 & 0.6970 & 2.2275 & 1.5593 \\
& 2 & 1.9943 & 3.9687 & 2.3388 \\
& 3 & 1.9954 & 3.9733 & 3.7581 \\
$90 / 0$ & 1 & 0.6970 & 2.2274 & 1.5594 \\
& 2 & 1.9944 & 3.9688 & 2.3393 \\
& 3 & 1.9957 & 3.9733 & 3.7580 \\
\hline
\end{tabular}

Table 11

Non - dimensional buckling loads $\bar{P}=P a^{2} / E_{1} h^{3}$ of (45/ -45) and (-45/45) lamination schemes of square laminated plates with $a / h=20$, and $E_{1} / E_{2}=40$

\begin{tabular}{ccccc}
\hline \multirow{2}{*}{$\begin{array}{c}\text { Lamination } \\
\text { order }\end{array}$} & Mode & \multicolumn{3}{c}{ Boundary Conditions } \\
\cline { 3 - 5 } & Number & SS & CC & CS \\
\hline \multirow{3}{*}{$45 /-45$} & 1 & 0.8375 & 1.6524 & 1.2806 \\
& 2 & 1.7263 & 2.7630 & 1.9965 \\
& 3 & 1.7285 & 2.7659 & 2.5358 \\
$-45 / 45$ & 1 & 0.8372 & 1.6527 & .2805 \\
& 2 & 1.7262 & 2.7631 & 19963 \\
& 3 & 1.7283 & 2.7660 & 2.5355 \\
\hline
\end{tabular}

Table 12

Non - dimensional buckling loads $\overline{\mathrm{P}}=\mathrm{Pa}^{2} / \mathrm{E}_{1} \mathrm{~h}^{3}$ of $(45 /-45)$ and $(-45 / 45)$ lamination schemes of square laminated plates with $\mathrm{a} / \mathrm{h}=20$, and $\mathrm{E}_{1} / \mathrm{E}_{2}=5$

\begin{tabular}{ccccc}
\hline \multirow{2}{*}{$\begin{array}{c}\text { Lamination } \\
\text { order }\end{array}$} & Mode & \multicolumn{3}{c}{ Boundary Conditions } \\
\cline { 3 - 5 } & Number & SS & CC & CS \\
\hline \multirow{3}{*}{$45 /-45$} & 1 & 0.9907 & 2.2010 & 1.6553 \\
& 2 & 2.1995 & 3.7613 & 2.5668 \\
& 3 & 2.2015 & 3.7652 & 2.4640 \\
$-45 / 45$ & 1 & 0.9908 & 2.2010 & 1.6553 \\
& 2 & 2.1995 & 3.7613 & 2.5671 \\
& 3 & 2.2015 & 3.7652 & 3.4636 \\
\hline
\end{tabular}

\subsection{Effect of Boundary Conditions}

The type of boundary support is an important factor in determining the buckling loads of a plate along with other factors such as aspect ratio, modulus ratio, ... etc.

Three sets of boundary conditions, namely simply - simply supported (SS), clamped - clamped (CC), and clamped - simply supported (CS) were considered in this study.

The variations of buckling load, $\bar{P}$ with the mode number for thin $(a / h=20)$ symmetrically loaded laminated cross - ply $(0 / 90 / 90 / 0)$ plate with modulus ratio $\left(E_{1} / E_{2}=5\right)$ were computed and the results are given in Table 13 and Figure 14.

It is observed that, for all cases the buckling load increases with the mode number but at different rates depending on whether the plate is simply supported, clamped or clamped - simply supported. The buckling load is a minimum when the plate is simply supported and a maximum when the plate is clamped. Because of the rigidity of clamped boundary condition, the buckling load is higher than in simply supported boundary condition. It is also observed that as the mode number increases, the plate needs additional support. 
Table 13

The first five non - dimensional buckling loads $\overline{\mathrm{P}}=\mathrm{Pa}^{2} / \mathrm{E}_{1} \mathrm{~h}^{3}$ of symmetric $(0 / 90 / 90 / 0)$ square laminated plates with $\mathrm{a} / \mathrm{h}=20$, and $\mathrm{E}_{1} / \mathrm{E}_{2}=5$

\begin{tabular}{cccc}
\hline Mode & \multicolumn{3}{c}{ Boundary Conditions } \\
\cline { 2 - 4 } Number & SS & CC & CS \\
\hline 1 & 0.6972 & 2.1994 & 1.8225 \\
2 & 1.2552 & 2.5842 & 2.0097 \\
3 & 2.4284 & 4.1609 & 2.7116 \\
4 & 2.6907 & 4.7431 & 4.3034 \\
5 & 2.7346 & 5.0168 & 4.4536 \\
\hline
\end{tabular}

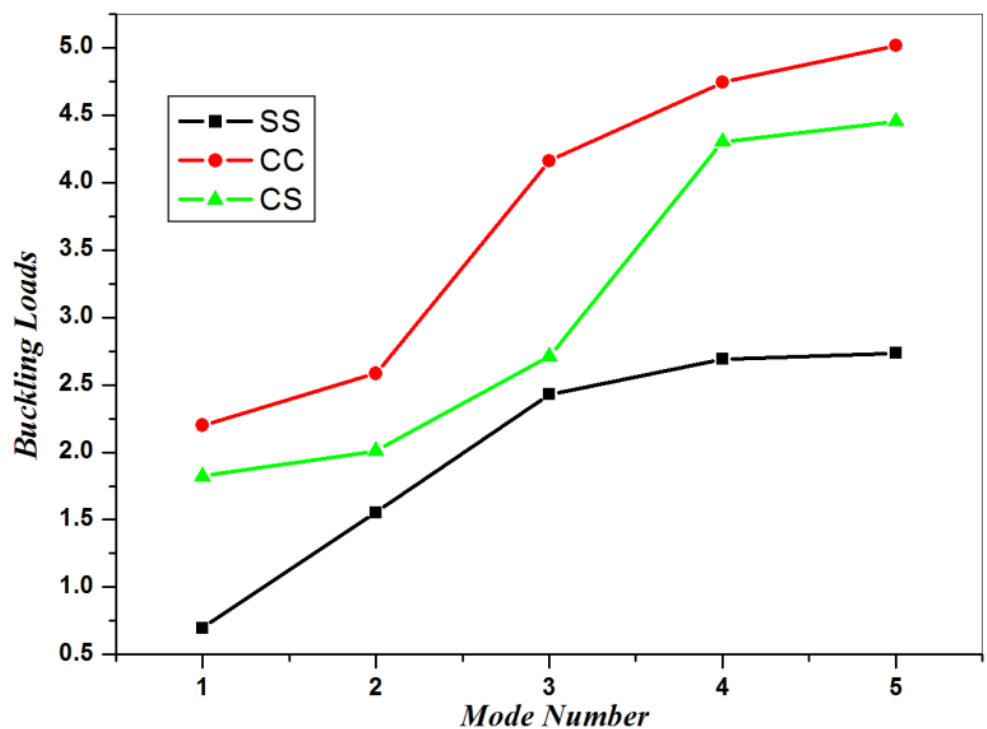

Figure 14. Effect of boundary conditions

\section{Conclusion}

The finite element model has been formulated to compute the buckling loads of laminated plates with rectangular cross - section and to study the effects of lamination scheme, aspect ratio, material anisotropy, fiber orientation of layers, reversed lamination scheme and boundary conditions on the non - dimensional critical buckling loads of laminated composite plates. Finally, a series of new results have been presented.

The symmetric laminate is stiffer than the anti-symmetric one. This phenomenon is caused by coupling between bending and stretching which lowers the buckling loads of symmetric laminate.

The buckling load is highly influenced by the end support. The buckling load of the quasi - isotropic $(0 /+45 /-$ 45/90) rectangular composite plate with clamped - clamped type boundary condition is 1.5 times higher than the buckling load of the composite plate with clamped - simply supported (CS) type boundary condition, and more than 3 times of simply - simply supported (SS) type boundary condition.

The buckling load increases continuously with increasing aspect ratio, but the rate of increase is not uniform. This may be due to the effect of bending - extensional twisting stiffness which increases the critical load.

As the plate becomes more restrained, its resistance to buckling increases. The reason is that the structural stiffness reduces due to its constraints.

The buckling load decreases with increase in modulus ratio. It is also observed that the variation of buckling load becomes almost constant for higher values of elastic modulus. This may be attributed to the coupling effect which increases with the increasing degree of anisotropy.

Suleiman, O. M. E., Osman, M. Y., \& Hassan, T. (2019). Stability of thin laminated decks plates under plane compressive loading. International Research Journal of Engineering, IT \& Scientific Research, 5(2), 1-28. 
The curves of simply - simply supported (SS) boundary conditions show maximum value of buckling load at $\theta=$ $45^{\circ}$. However, this trend is different for a plate under clamped - clamped (CC) boundary conditions which show minimum load at $\theta=45^{\circ}$. For clamped - simply supported, it is observed that the buckling load decreases continuously with $\theta$. This may be due to the total and partial fixed rotation $\phi$ and $\psi$ in the two later cases.

The buckling load of laminated plates will remain the same even if the lamination order is reversed. The reason behind this is that the transformed elastic coefficients, $\left[\mathrm{C}_{\mathrm{ij}}\right]$, are equal for both lamination schemes.

The buckling load increases with the mode number but at different rates depending on whether the plate is simply supported (SS), clamped (CC) or clamped - simply supported. The buckling load is a minimum when the plate is simply supported and a maximum when the plate is clamped. Because of the rigidity of clamped boundary condition, the buckling load is higher than in simply supported boundary condition. It is also observed that as the mode number increases, the plate needs additional support.

Conflict of interest statement and funding sources

The authors declared that they have no competing interest. The study was financed by personal funding.

Statement of authorship

The authors have a responsibility for the conception and design of the study. The authors have approved the final article.

\section{Acknowledgments}

The authors would like to acknowledge with deep thanks and profound gratitude Mr. Osama Mahmoud of Daniya Center for Publishing and Printing Services, Atbara, Sudan who spent many hours in editing, re - editing of the manuscript in compliance with the standard format of IRJEIS. 


\section{References}

Bao G., Jiang W., Roberts J.C.,' Analytic and finite element solutions for bending and buckling of orthotropic rectangular plates', International Journal of solids and structures; (1997),34(14): PP. (1792 - 1821).

Bazant Z.P., Gedolin L.,' Stability of structures', New York: Oxford University press; (1991).

Berthelot J.M.,' Composite materials mechanical behavior and structural analyses', New York: Springer; (1999).

Bert C.W., Malik M.,' Differential quadrature: A powerful new technique for analysis of composite structures', composite structures; (1997), 39(3-4): PP. (179 - 189).

Bhimaraddi A., Stevens L.K.,' A higher - order theory for free vibration of orthotropic, homogeneous, and laminated rectangular plates', Journal of applied mechanics; (1984), 51: PP. (195 - 198).

Bert C.W.,'A critical evaluation of new plate theories applied to laminated composites', Composite structures; (1984),2: PP. (329 - 347).

Chai G.B., Khong P.W.,' The effect of varying support conditions on the buckling of laminated composite plates', Composite structures; (1993), No.24(2): PP. (99 - 106).

Chang C.C., Ebcioglu I.K., Haight C.H.,' General stability analysis of orthotropic sandwich panels for four different boundary conditions', Zeitschr Angew, Math. Mech.; (1962), 43: PP. (373 - 389).

Cho M., Parmerter R.R.,' Efficient higher - order composite plate theory for laminated composites', AIAA Journal; (1993), 31 - 7: PP. (1299-1306).

Cook R.D.,' Concepts and applications of finite element analyses', New York: Wiley; (1981).

Dawe D.J., Wang S.,' Spline finite strip analysis of the buckling and vibration of rectangular composite laminated plates', International Journal of mechanical sciences; (1995),37(6): PP. (645 - 667).

Di sciuva M.,' An improved shear deformation theory for moderately thick multi - layered anisotropic shells and plates', Journal of applied mechanics; (1987), 54: PP. (589-596).

Eisenberger M., Alexandrov A.,' Buckling loads of variable thickness thin isotropic plates', thin - walled structures; (2003), 41(9): PP. (871 - 889).

Fares M.E., Zenkour A.M.,' Buckling and vibration of non - homogeneous composite cross - ply laminated plates with various plate theories', Composite structures; (1999), 44: PP. (279-287).

Gilat R., Williams T.O., Aboudi J.,' Buckling of composite plates by global - local plate theory', Composite part B Engineering; (2001), 32: PP. (229 - 236).

GU H., Chattopadhyay A.,' Three - dimensional elasticity solution for buckling of composite laminates', Composite structures; (2000), 50: PP. (29-35).

Hadian J., Nayfeh A.H,' Free vibration and buckling of shear deformable cross - ply laminated plates using state space concept', Computer and structures;(1993),4: PP. (677 - 693).

Huang Y.Q., Li Q.S.,' Bending and buckling analysis of anti - symmetric laminates using the least square differential quadrature method', Computer methods in applied mechanics and engineering, 193; (2004): PP. (3471 - 3492).

Iyengar N.G.,' Structural stability of columns and plates', Chichester, England: Ellis Horwood limited publishers; (1988).

Jiang W., Bao G., Roberts J.C.,' Finite element modeling of stiffened and unstiffened orthotropic plates', Computer and structures Journal; (1977), 63(1): PP. (105 - 117).

Jones R.M.,' Buckling and vibration of unsymmetrical laminated cross - ply rectangular plates', AIAA Journal; (1973), 12(11) : PP. (1626 - 1632).

Kant T., Pandya,' A simple finite element formulation of higher - order theory for unsymmetrical laminated composite plates', Composite structures; (1988), 98: PP. (215 - 224).

Kant T., Kommineni J.R.,' geometrically non - linear analysis of symmetrically laminated composite and sandwich shells with higher - order theory and Co finite elements', composite structures; (1984), 27: PP. (403 - 418).

Karama M., Afaqk S., Mistou S.,' Mechanical behavior of laminated composite beam by the new multi - layered laminated composite structures model with transverse shear stress continuity', International Journal of solids and structures;(2003),40: PP. (1525 - 1546).

Kerr A.D.,' An extended Kantorovich method for solution of Eigen value problem', International Journal of solids and structures; (1969), 5 (7): PP. (559- 572).

Khdeir A.A.,' Free vibration and buckling of unsymmetrical cross - ply laminated plates using a refined theory', Journal of sound and vibration; (1989), 128: PP. (377 - 395).

Kim Y.S., Hoa S.V.,' biaxial buckling behavior of composite rectangular plates', composite structures; (1995), 31(4): PP. $(247-252)$.

Suleiman, O. M. E., Osman, M. Y., \& Hassan, T. (2019). Stability of thin laminated decks plates under plane compressive loading. International Research Journal of Engineering, IT \& Scientific Research, 5(2), 1-28. 
Leissa A.W., Kang J.,' Exact solutions for vibration and buckling of an SS - C - SS - C rectangular plate loaded by linearly varying in - plane stresses', International Journal of mechanical sciences;(2002),44: PP. (1925 - 1945).

Leissa A.W.,' Conditions for laminated plates to remain flat under in - plane loading', Composite structures; (1986), 6: PP. $(261-270)$.

Levinson M.,' an accurate simple theory of the statics and dynamics of elastic plates', Mechanical research community; (1980), 7(6): PP. (343 - 350).

Librescu L.,' Electrostatics and kinetics of anisotropic and heterogeneous shell - type structures', Noordhoff: Leyden; (1975).

Liu G.R., Chen X.L., Reddy J.N.,' Buckling of symmetrically laminated composite plates using the element free Galerkin method', International Journal of structural stability dynamics; (2002), 2(3): PP. (281 - 294).

Lo K.H., Christensen R.M., Wu E.M.,'A high - order theory of plate deformation', part 1: homogeneous plates, Journal of applied mechanics, Trans. ASME; (1977), 44(4): PP. (663 - 668).

Mallikarjuna, Kant T.,' A critical review and some results of recently developed refined theories of fibers reinforced laminated composites and sandwich plates', Composite structures; (1993),23: PP. (293 - 312).

March H.W., Smith C.B.,' Buckling loads of flat sandwich panels in compression', Forest products research laboratory report No.1525, Madison, WI; (1945).

Matsunaga H.,' Vibration and stability of cross - ply laminated composite plates according to a global higher - order plate theory', composite structures; (2000), 48: PP. (231 - 244).

Mindlin R.D.,' Influence of rotatory inertia and shear on flexural motions of isotropic elastic plates', Trans. ASME Journal of applied mechanics; (1951), 18: PP. (31 - 38).

Murthy M.V.V.,' an improved transverse shear deformation theory for laminated anisotropic plates', NASA technical paper, 1903; (1981) : PP. (1 - 37).

Narita Y., Fukushi K.,' Analysis program for generally laminated rectangular plates based on classical plate theory', Trans. J. SME, (A); (1996), PP. (2621 - 2627).

Narita Y., Leissa W.L.,' Buckling studies for simply supported symmetrically laminated rectangular plates', Composite structures; (1989), 12: PP. (113 - 132).

Naruoka M., 'Bibliography on theory of plates', Gihodo, Tokyo; (1981).

Noor A.K.,' Stability of multi - layered composite plates', Fiber science technology; (1975), 8: PP. (81 - 89).

Noor A.K., Burton W.S.,' Assessment of computational models for multi - layered anisotropic plates', Composite structures; (1990), 14: PP. (233 - 265).

Osama Mohammed Elmardi Suleiman, 'Introduction and Literature Review on Buckling of Composite Laminated Plates', Lap Lambert Academic Publishing, Germany, and ISBN: (978-3-659-86387-5) ;(March 2016).

Osama Mohammed Elmardi Suleiman, 'Bibliography and Literature Review on Buckling of Laminated Plates', International Journal of Science and Engineering, volume 2, issue 8; (August 2016), pp. (104 - 112).

Pagano N.J.,' Exact solution for rectangular bi - directional composite and sandwich plates', Journal of composite materials; (1970), 4: PP. $(20-34)$.

Phan N.D. and Reddy J.N., 'Analysis of laminated composite plate using higher - order shear deformation theory', International Journal of numerical methods in engineering, vol.21; (1985): pp. (2201-2219).

Qatu M.S., Leissa A.W.,' Buckling of transverse deflections of unsymmetrically laminated plates subjected to in plane loads', AIAA Journal; (1993),31(1): PP. (189 - 194).

Reddy J.N.,' A refined non - linear theory of laminated plates with transverse shear deformation', International Journal of solids and structures; (1984), 51: PP. (319-330).

Reddy J.N.,' an evaluation of equivalent single layer and layer wise theories of composite laminates', Composite structures; (1993), 25: PP. (21-35).

Reddy J.N.,' On refined theories of composite laminates', Mechanical; (1980), 25(4): PP. (230 - 238$).$

Reddy J.N., Robbins Jr D.H.,' Theories and computational models for composite laminates', Applied mechanics review, part 1; (1994), 47(6): PP. $(147-165)$.

Reddy J.N., second edition,' Mechanics of laminated composite plates and shells', Boca Raton CRC press; (2004).

Reddy J.N.,' Mechanics of laminated composite plates: Theory and Analyses', Boca Raton: CRC press; (1997).

Reddy J.N.,' Energy and variational methods in applied mechanics', New York: Wiley; (1984).

Reddy J.N., Phan N.S.,' Stability and natural vibration of isotropic, orthotropic and laminated plates according to a higher order shear deformable theory', Journal of sound and vibration; (1985), 9 (82): PP. (157 - 170).

Reddy J.N., Khdeir A.A.,' Buckling and vibration of laminated composite plates using various plate theories', AIAA; (1989), 27 (12): PP. (1808 - 1817). 
Robinson J.R.,' The buckling and bending of orthotropic sandwich panels with all edges- simply supported', Aero Q; (1955), 6(2): PP. (125).

Schmidt R.,' A refined non - linear theory of plates with transverse shell deformation', Journal of India mathematical society; (1977), 27(1): PP. (23 - 38).

Seide P.,' an improved approximate theory for the bending theory of laminated plates', Mechanics today; (1980), 5: PP. (451-466).

Shufrin I., Rabinovitch O., Eisenberger M.,' Buckling of symmetrically laminated rectangular plates with general boundary conditions - A semi analytical approach', composite structures; (2007).

Shufrin I., Eisenberger M.,' stability and vibration of shear deformable plates - first order and higher order analyses', International Journal of solid structures; (2005), 42(3 - 4): PP. (1225 - 1251).

Singer J., Arbocz J., Weller T.,' Buckling experiments: Experimental methods in buckling of thin walled strictures', vol.1, New York: John Willey and sons ;(1998).

Singer J., Arbocz J., Wetter T.,' buckling experiments: Experimental methods in buckling of thin walled structures: shells, Built up structures, composites and additional topics', vol.2., New York: John Willey and Sons; (2002).

Smith C.S.,' Design of marine structures in composite materials', Amsterdam: Elsevier science publishers Ltd; (1990).

Soladatos K.P.,' a transverse shear deformation theory for homogeneous monoclinic plates', Acta Mech.; (1992), 94: PP. $(195-220)$.

Touratier M.,' An efficient standard plate theory', International Journal of engineering sciences; (1991), 29(8): PP. $(901-916)$.

Turvey G.J., Marshall I.H.,' Buckling and post buckling of composite plates', London: Chapman and Hall; (1995).

Ungbhakorn V., Singhatanadgid P.,' Buckling analysis of symmetrically laminated composite plates by extended Kantorovich method', Composite structures; (2006); 73(1): PP. (120 - 128).

Whitney J.M.,' Structural analysis of laminated anisotropic plates', Lancaster, PA: Technomic Publishing; (1987).

Yang H.T.Y., Saigal S., Masud A., Kapania R.K.,' A survey of recent shell element', International Journal for numerical methods in engineering,47 (1-3); (2000): PP. (101 - 127).

Yuan S., Jin Y.,' Computation of elastic buckling loads of rectangular thin plates using the extended Kantorovich method', computer structures; (1998), 66(6): PP. (861 - 867).

Zienkiewicz O.C.,' The finite element method', England: McGraw Hill; (1977).

Suleiman, O. M. E., Osman, M. Y., \& Hassan, T. (2019). Stability of thin laminated decks plates under plane compressive loading. International Research Journal of Engineering, IT \& Scientific Research, 5(2), 1-28. 
Biography of Author

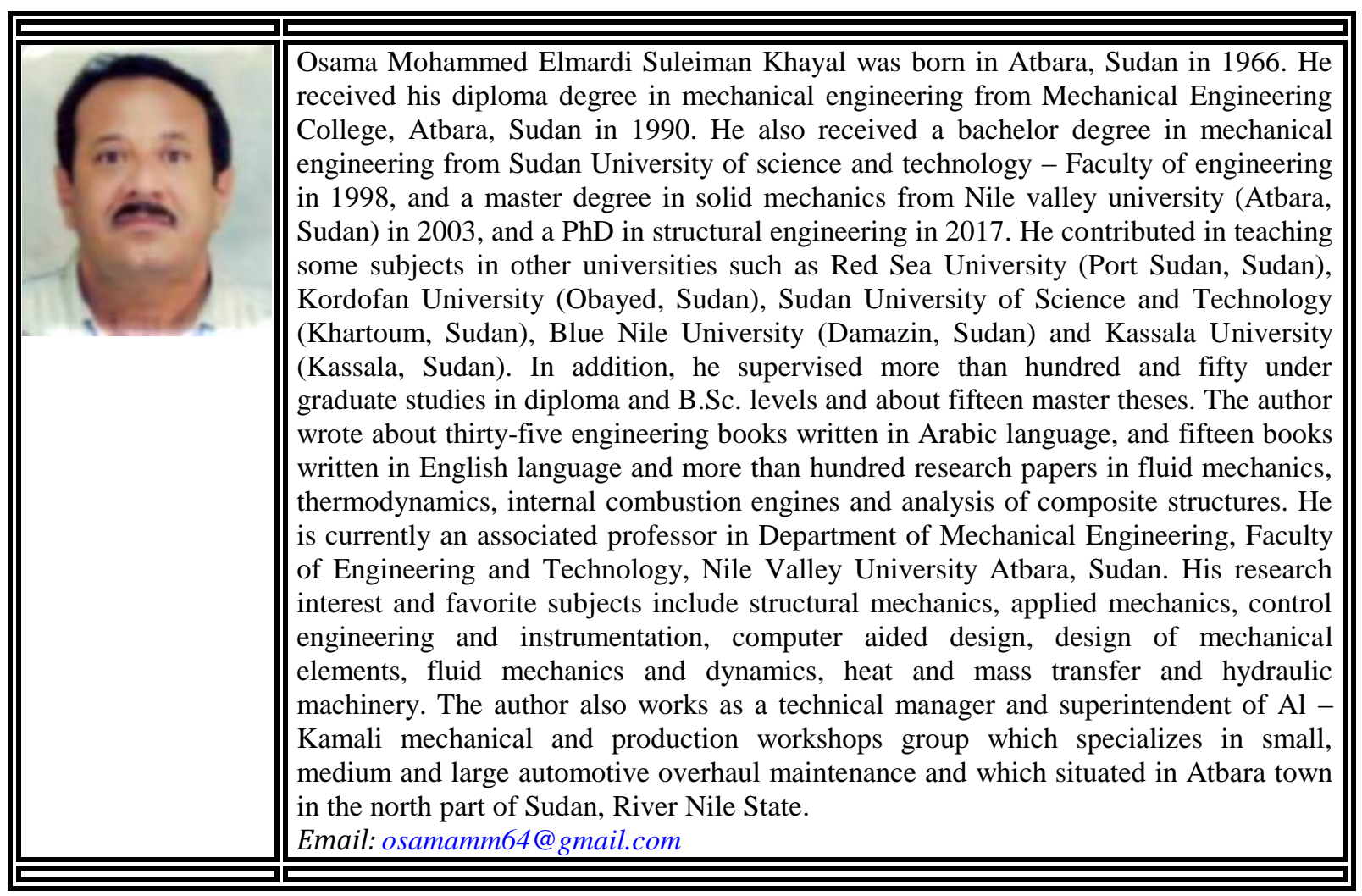

